\begin{abstract}
This paper investigates the statistical properties of the Black-Scholes option price under a Bayesian approach. We incorporate randomness both in the price process and in volatility to derive the prior and posterior densities of a European call option. Expressions for the density of the option price conditional on the sample estimate of volatility and on the asset price respectively, are also derived. Numerical results are presented to compare how the dispersion of the option price changes in the transition from prior to posterior information, where information may be price or sample variance or both. The derived expression for the posterior density is of considerable interest since it can be straightforwardly combined with a loss function to produce optimal estimates of options prices.
\end{abstract}

Keywords: Bayes, Black-Scholes, option price, posterior density, volatility. JEL Classification: C11, G13. 


\title{
Bayesian Analysis of the Black-Scholes Option Price
}

\author{
Theofanis Darsinos ${ }^{1}$ and Stephen Satchell \\ Faculty of Economics \& Politics \\ University of Cambridge
}

January 20, 2001

\section{Introduction}

The focal point of a great deal of econometric work within the framework of option valuation has long been the problem of estimating the parameters of continuous-time price processes which act as inputs for parametric derivative pricing models. Since the volatility of the asset price is conditionally the only unobservable and potentially stochastic component entering the Black-Scholes (1973) formula, attempts of academics and practitioners to improve on their estimates of option prices have generally focused on the issue of volatility modelling. From the simple models of estimation from historical price and return data (e.g. maximum likelihood using continuously compounded returns calculated from closing prices, Parkinson's (1980) "extreme value method" incorporating high-low prices, Garman and Klass (1980) adding opening prices, etc.) to ARIMA modelling of the time-series behaviour of volatility (Poterba and Summers (1986), French, Schwert and Stambaugh (1987)), ${ }^{2}$ and from the $(\mathrm{G}) \mathrm{ARCH}$ class of stationary conditionally-heteroscedastic processes implicitly allowing for the conditional variance to be time-varying (Engle (1982), Bollerslev (1986), Engle and Bollerslev (1987), Nelson (1991), Day and Lewis (1992), Engle and Mustafa (1992), and many others) to stochastic variance models (Hull and White (1987), Scott (1991), Wiggins (1987), Melino and Turnbull (1990), etc), to implied volatility approaches (Latane and Rendleman (1976), Chiras and Manaster (1978), Day and Lewis (1988)). The plethora of practices is overwhelming indeed.

It is not the aim of this paper to antagonise this vast literature with yet another volatility predictor. Rather our scope is to suggest an option price predictor by providing a Bayesian analysis of the Black-Scholes option price (BS hereafter), where randomness in the option price arises from randomness in the volatility of returns and the stock price process. The BS price as an unconditional random variable depends on both the aforementioned arguments while as a conditional variable depends only on

\footnotetext{
${ }^{1}$ Financial support from the A.G. Leventis Foundation, the Wrenbury Scholarship Fund at the University of Cambridge, and the ESRC is gratefully acknowledged.

${ }^{2}$ These models act as approximations to a slowly changing time varying volatility.
} 
the former. One should therefore combine a prior density for the (price, volatility) vector together with the likelihood of volatility to obtain the posterior density of price and volatility. The posterior density of the option price then follows after dividing by the marginal density of the asset price and applying a non-linear transformation. What is appealing with our Bayesian approach, is that it allows us to account for randomness both in the price process and in volatility, something that has been neglected in the previous literature since only the conditional nature of the formula has been investigated.

Bayesian methods have been used in the past to model the variance of stock returns for the purpose of option valuation. Karolyi (1993) utilises ${ }^{3}$ prior information extracted from the cross sectional patterns in the return volatilities for groups of stocks sorted either by size, -or financial leverage, -or trading volume, together with the sample information, to derive the posterior density of the variance. He reports improved prediction accuracy for estimates of option prices calculated using the Bayesian volatility estimates relative to those computed using implied volatility, standard historical volatility, or even the actual ex-post volatility that occurred during each option's life. We find this result interesting both on its own ground and as a motivation to use a Bayesian approach to explore the statistical properties of the BS option price.

Karolyi also suggests that the posterior density of the option price can be derived as a non-linear transformation of that of the stock return volatility. We extend his analysis by considering options in terms of price and volatility. This is because prior to sampling the option value depends on both prices and volatility and this should be taken into account when deriving the posterior. Moreover, it is much more appealing for forecasting options prices out-of-sample, to allow both prices and volatility to vary, as opposed to only volatility.

Ncube and Satchell (1997), investigate the properties of the BS price under the classical approach. They, take advantage of the monotonicity properties of the option price with respect to the asset price and volatility, to obtain the conditional distribution of what they call the "true" BS price as well as the conditional distribution of what they call the "predicted" BS price. The former is obtained by conditioning on volatility (they assume that volatility is known and not estimated), while the latter follows from conditioning on the underlying asset price and treating the only source of randomness as being due to the classical variance estimate. This approach however, is not the most realistic one; Ncube and Satchell's so-called "true" BS price stands on the unrealistic assumption that volatility is known and not estimated. We offer a much more orthodox alternative, namely the unconditional (prior) distribution of the BS price. Turning to the "predicted" BS price, our posterior distribution is more informationaly efficient and is theoretically favourable to produce better estimates of option prices.

\footnotetext{
${ }^{3}$ as has been suggested by Black (1976), Christie (1982), Epps and Epps (1976), Morgan (1976), Tauchen and Pitts (1983))
} 
Epitomising, in this paper we account for randomness in price and volatility to provide a full Bayesian analysis of the BS option price. As a Bayesian problem, the randomness of the BS price is unusual in that it depends both on data (price) and parameters (volatility). Under the model's assumptions (i.e. log-normality of stock prices) we derive the prior and posterior densities for a European call. We also provide expressions for the density of a call option conditional on the sample estimate of volatility and conditional on the asset price respectively. To this end, we investigate how the dispersion of the option price changes as we condition on more information: from the prior density, to conditioning only on the sample variance, to conditioning on the price, to the posterior density. The results we present, for a number of realistic values, show the extent to which conditioning on the asset price dramatically reduces the variability of the option price.

Our paper could be criticised on the grounds that option pricing has moved a long way from the BS model. Our response is that the BS model is still widely used in applications, especially in real options. For a detailed list of applications (e.g. in real options, bankruptcy problems, evaluation of insured bank deposits, actuarial work etc.) see Knight and Satchell (1997). The organisation of the paper is as follows. In Section 2 we present the main theory: Subsection 2.1 introduces the stochastic model assumed to generate the data and sets up a Bayesian framework. In Subsections 2.2, and 2.3 we work towards deriving the prior and posterior densities of the option price. Section 3 deals with their numerical evaluation. In Section 4 we present our results and Section 5 concludes.

\section{Derivation of the prior and posterior densities for the Black-Scholes option price.}

\subsection{Distributional assumptions.}

In parametric derivative pricing models, such as the BS, the price process of the underlying asset is fully specified up to a finite number of unknown parameters. ${ }^{4}$ Here we use the traditional log-normal diffusion with unknown drift and volatility. It is therefore assumed that in the continuous-time limit the asset price at time $t$ is $P_{t}$ where $P_{t}$ is determined by the stochastic differential equation:

$$
d P_{t}=\mu P_{t} d t+\sigma P_{t} d W_{t}
$$

\footnotetext{
${ }^{4}$ This is in contrast to non-parametric derivative pricing models, where the price process is not explicitly specified but is rather inferred from the data under suitable regularity conditions; (see for example Hutchinson, Lo, and Poggio (1994), Rubinstein (1994), Jackwerth and Rubinstein (1996)).
} 
with $\mu$ the expected rate of return, $\sigma$ the volatility, and $\left\{W_{t}, t \geq 0\right\}$ a standard Brownian motion. Then the asset price process may be represented as:

$$
P_{t}=P_{0} \exp \left[\left(\mu-\frac{\sigma^{2}}{2}\right) t+\sigma\left(W_{t}-W_{0}\right)\right]
$$

The geometric return (continuously compounded return) for the stock between time 0 and $t$ is

$$
x_{t}=\log \left(P_{t} / P_{0}\right)
$$

From equation (2.1) it follows that the log-return $x_{t}$ is generated by an independent normal process with $x_{t} \sim N\left(\left(\mu-\sigma^{2} / 2\right) t, \sigma^{2} t\right) .^{5}$ This then implies that $\log P_{t} \sim N\left(\log P_{0}+\left(\mu-\frac{1}{2} \sigma^{2}\right) t, \sigma^{2} t\right)$.

ASSUMPTION 2.1. The asset price $P_{t}$ is log-normally distributed. Its conditional probability density function is given by: ${ }^{6}$

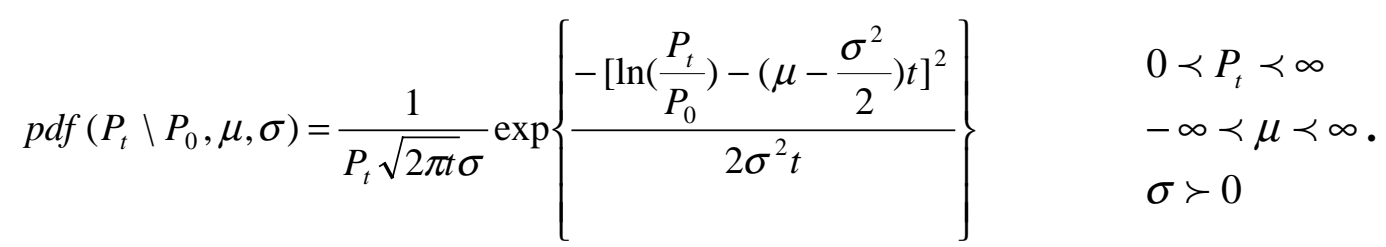

ASSUMPTION 2.2. When the variance $\sigma^{2}$ of an independent normal process is assumed known but the mean $\mu$ is a random variable, the most convenient distribution for $\mu$ (the natural conjugate of the likelihood of the sample) is the normal distribution. The conditional probability density function of the expected rate of return $\mu$ is therefore given by:

$$
p d f(\mu \backslash \sigma)=\frac{\sqrt{t}}{\sqrt{2 \pi} \sigma} \exp \left(-\frac{t(\mu-m)^{2}}{2 \sigma^{2}}\right) \quad \begin{gathered}
-\infty \prec \mu, m \prec \infty, \\
\sigma \geq 0, t \succ 0
\end{gathered}
$$

where $m$ is a hyperparameter.

\footnotetext{
${ }^{5} N(\ldots)$ denotes the normal distribution.

${ }^{6}$ Here and below we use $p d f$ to denote probability density functions generally and not one specific probability density. The argument of $p d f$ as well as the context in which it is used will identify the particular $p d f$ being considered.
} 
Since the log-return $x$ between two consecutive time intervals is normally distributed with variance $\sigma^{2}$, the classical minimum-variance unbiased estimator of $\sigma^{2}$ for $t$ observations is

$$
s^{2}=\sum_{i=1}^{t}\left(x_{i}-\bar{x}\right)^{2} /(t-1)
$$

where $\bar{x}=(1 / t) \sum_{j=1}^{t} x_{j}$

It is well known that the statistic $(t-1) s^{2} / \sigma^{2}$ has a $\chi^{2}$ distribution with $t-1$ degrees of freedom. This in turn implies that the estimator $s^{2}$ is distributed $\sigma^{2} \chi^{2} /(t-1)$ with $t-1$ degrees of freedom.

ASSUMPTION 2.3. The likelihood function for $\sigma^{2}$ is therefore defined as: ${ }^{7}$

$$
L\left(\sigma^{2} \backslash s^{2}\right) \equiv f_{\chi 2}\left(\frac{(t-1) s^{2}}{\sigma^{2}}\right) \frac{t-1}{\sigma^{2}}
$$

$\Rightarrow \quad p d f\left(s^{2} \backslash \sigma^{2}\right)=\left(\frac{t-1}{2}\right)^{\frac{t-1}{2}} \frac{\left(s^{2}\right)^{\frac{(t-1)}{2}-1}}{\Gamma\left(\frac{t-1}{2}\right)\left(\sigma^{2}\right)^{\frac{t-1}{2}}} \exp \left(-\frac{(t-1) s^{2}}{2 \sigma^{2}}\right)$.

or $\quad p d f(s \backslash \sigma)=\frac{2\left(\frac{v}{2}\right)^{\frac{v}{2}}}{\Gamma\left(\frac{v}{2}\right)} \frac{s^{v-1}}{\sigma^{v}} \exp \left(-\frac{v s^{2}}{2 \sigma^{2}}\right) \quad$ where $v=t-1$.

The fact that $(t-1) s^{2} / \sigma^{2} \sim \chi^{2}{ }_{(t-1)}$, suggests that the conditional probability density function for the variance (i.e. $p d f\left(\sigma^{2} \backslash s^{2}\right)$ ) is Inverted-Gamma-1. We use this as motivation when choosing the prior density for $\sigma^{2}$.

ASSUMPTION 2.4. We can assign an Inverted-Gamma-1 distribution with hyperparameters $\lambda, \theta$ as the prior distribution for $\sigma^{2}$. Its prior probability density function is then given by: ${ }^{8}$

${ }^{7} f_{\chi 2}(\ldots)$ denotes the $\chi^{2}$ probability density function.

${ }^{8} f_{i \gamma}(\ldots)$ denotes the Inverted-Gamma-1 probability density function. 


$$
\begin{gathered}
\operatorname{pdf}\left(\sigma^{2} \backslash \lambda, \theta\right)=f_{i \gamma}\left(\sigma^{2} \backslash \lambda, \theta\right)=\frac{\lambda^{\theta}}{\Gamma(\theta)} \frac{1}{\left(\sigma^{2}\right)^{\theta+1}} \exp \left(-\frac{\lambda}{\sigma^{2}}\right) \\
\text { or: } \quad p d f(\sigma \backslash \lambda, \theta)=2 \sigma \frac{\lambda^{\theta}}{\Gamma(\theta)} \frac{1}{\left(\sigma^{2}\right)^{\theta+1}} \exp \left(-\frac{\lambda}{\sigma^{2}}\right) \quad \lambda, \theta \succ 0 .
\end{gathered}
$$

A Bayesian framework has now been introduced. Assumptions 2.2 and 2.4 define prior distributions for the process parameters $\mu$ and $\sigma$. Assumption 2.3 defines the likelihood for the variance (volatility). The process for the stock price is represented in equation (2.2) while the conditional probability density function for the stock price is defined in Assumption 2.1.

Karolyi (1993) provided a Bayesian analysis for the stock return volatility. He combined an Inverted-Gamma prior density (i.e. $\left.p d f\left(\sigma^{2} \backslash \vartheta\right)\right)^{9}$ together with the likelihood (i.e. $\left.L\left(\sigma^{2} \backslash s^{2}, t\right)\right)$ to obtain the posterior probability density function of the variance (i.e. $\left.\operatorname{pdf}\left(\sigma^{2} \backslash s^{2}, t ; \vartheta\right)\right)$.

\subsection{The prior density.}

For $0 \leq t \leq T$, the time- $t$ price of a European call option at strike price $K$ with expiry time $\tau=T-t$ is:

$$
c=C\left(P_{t}, K, \sigma, \tau, r\right)
$$

$$
=P_{t} \Phi\left(\frac{\log \left[\frac{P_{t}}{K}\right]+\left[r+\frac{1}{2} \sigma^{2}\right] \tau}{\sigma \sqrt{\tau}}\right)-K \exp (-r \tau) \Phi\left(\frac{\log \left[\frac{P_{t}}{K}\right]+\left[r-\frac{1}{2} \sigma^{2}\right] \tau}{\sigma \sqrt{\tau}}\right)
$$

where $r$ is the risk-free interest rate (assumed fixed and known from 0 to $T$ ) and everything else as already defined.. The term $\Phi(\cdot)$ denotes the standard normal cumulative distribution function. We shall either condition on time $t$ or on time 0 information, and the latter we shall refer to as unconditional.

REMARK 2.1. The information available at time $t$ is the history of the discrete price process, $\left(P_{0}, P_{1}, P_{2}, \ldots, P_{t}\right)$. Then conditionally on time $t$, randomness in the option price stems only from the unknown volatility $\sigma$. One may therefore write $c=C(\sigma)$.

\footnotetext{
${ }^{9} \vartheta$ is a 2-dimensional vector of prior parameters estimated using information extracted from the crosssectional patterns in return volatilities for groups of stocks sorted on size, financial leverage, and trading volume.
} 
As aforementioned, literature on the estimation of $\sigma^{2}$ abounds. The benchmark procedure is to use the classical estimator $s^{2}$ given in (2.4). The paper by Boyle and Ananthanarayanan (1977) first evaluates the impact of variance estimation in option valuation models. The authors recognise that using $s^{2}$ as an estimate of the variance does produce biased option prices. ${ }^{10}$ However they claim that the magnitude of the bias is not large and are more concerned with the dispersion induced in the option price. Interestingly, they suggest that a Bayesian approach may be usefully employed to improve on the precision of option price estimates.

Butler and Schachter (1986) on the other hand, are concerned with the varianceinduced option price bias and investigate potential remedial measures. In fact they construct a uniformly minimum variance unbiased estimator for the BS option price. The estimator is derived by taking a Taylor series expansion of the pricing formula and the moments of the estimated variance.

In a discussion of the Butler and Schachter paper, Knight and Satchell (1997) reexamine the question of statistical bias in the BS option price. They show that the only unbiased estimated option is an at-the-money option. However, they argue that the importance of bias in option pricing seems minor compared with other obvious sources of mispricing.

Noh, Engle, and Kane (1994) assess the performance of ARCH models for pricing options. Rather than comparing implied volatilities with GARCH volatilities, their study compares predictions of options prices from GARCH with predictions of the same options prices from forecasting implied volatility. The results indicate that both methods can effectively forecast prices well enough to profit by trading if transaction costs are not too high. The GARCH models are considerably more effective.

REMARK 2.2. Unconditionally, the option price depends both on the volatility $\sigma$ and the stock price process $P_{t}=P_{0} \exp \left[\left(\mu-\left(\sigma^{2} / 2\right)\right) t+\sigma\left(W_{t}-W_{0}\right)\right]$. We write $c=C\left(P_{t}, \sigma\right)$ to denote that fact. Bayesian theory mandates that this should be taken into account when deriving the posterior density. In other words we should treat prices and volatility as unknown random variables and identify a prior density for them.

The building block for the derivation of the prior density of the BS option price ${ }^{11}$ is therefore the joint density function of $P_{t}$ and $\sigma$; i.e. $p d f\left(P_{t}, \sigma \backslash P_{0}\right)$. Then by transforming $p d f\left(P_{t}, \sigma \backslash P_{0}\right)$ to $p d f\left(c, \sigma \backslash P_{0}\right)$ and integrating out $\sigma$ we obtain $p d f\left(c \backslash P_{0}\right)$. In what follows we shall ignore the dependence on $P_{0}$.

\footnotetext{
${ }^{10}$ This is because of the non-linearity of the option price formula.

${ }^{11}$ When we refer to the probability density of the BS option price we mean the probability density of a European call option.
} 
PROPOSITION 2.1. The joint unconditional density of $P_{t}$ and $\sigma$ is given by:

$$
p d f\left(P_{t}, \sigma\right)=\frac{1}{\sqrt{\pi t} P_{t}} \frac{\lambda^{\theta}}{\Gamma(\theta)} \frac{1}{\sigma^{2(\theta+1)}} \exp \left(-\frac{\lambda}{\sigma^{2}}\right) \exp \left(-\frac{1}{4 \sigma^{2} t}\left[\ln \left(\frac{P_{t}}{P_{0}}\right)-\left(m-\frac{1}{2} \sigma^{2}\right) t\right]^{2}\right)
$$

Proof. From Assumptions 2.4 and 2.2 we have $p d f(\sigma)$ and $p d f(\mu \backslash \sigma)$ respectively. Then $p d f(\mu, \sigma)=p d f(\sigma) * p d f(\mu \backslash \sigma)$. Similarly $p d f\left(P_{t}, \mu, \sigma\right)=$ $p d f(\mu, \sigma)^{*} p d f\left(P_{t} \backslash \mu, \sigma\right)$ with $p d f\left(P_{t} \backslash \mu, \sigma\right)$ given in Assumption 2.1. Finally $p d f\left(P_{t}, \sigma\right)=\int_{-\infty}^{\infty} p d f\left(P_{t}, \mu, \sigma\right) \partial \mu$. Section A.1 in the Appendix contains the analytic proof and all the relevant calculations.

We are now in a position to derive the unconditional density function of the option price. Let us first obtain $p d f(c, \sigma)$; take $p d f\left(P_{t}, \sigma\right)$ and consider the transformation:

$$
\begin{aligned}
& c=C\left(P_{t}, \sigma\right) \Rightarrow \Psi(c)=\Psi(c, \sigma)=P_{t} \\
& \sigma=\sigma
\end{aligned}
$$

where $\Psi$ is the inverse of the option price with respect to $P_{t} \cdot{ }^{12}$ The Jacobian $J$ of the transformation is given by:

$$
\frac{1}{J}=\left|\begin{array}{ll}
\frac{\partial c}{\partial P_{t}}=\Phi\left(d_{1}\right) & \frac{\partial c}{\partial \sigma}=v e g a \\
\frac{\partial \sigma}{\partial P_{t}}=0 & \frac{\partial \sigma}{\partial \sigma}=1
\end{array}\right|=\Phi\left(d_{1}\right)
$$

where $\quad d_{1}=\frac{\left[\ln \left(\frac{P_{t}}{K e^{-r \tau}}\right)+\frac{\sigma^{2} \tau}{2}\right]}{\sigma \sqrt{\tau}} \quad$ and $\quad$ vega $=\phi\left(\frac{\ln \left(\frac{P_{t}}{K e^{-r \tau}}\right)+\frac{\sigma^{2} \tau}{2}}{\sigma \sqrt{\tau}}\right) P_{t} \sqrt{\tau}$

$\phi(\ldots)=\Phi^{\prime}(\ldots)$ denotes the standard normal probability density function.

Then

\footnotetext{
${ }^{12}$ We invert the option pricing formula in terms of $P_{t}$, hence obtaining $P_{t}$ as a function of $c$ and $\sigma$. It should however be noted that there is no analytic expression (with the exception of an at-the-money option) for $P_{t}=\Psi(c, \sigma)$ and a Newton-Raphson numerical approximation is required.
} 


$$
p d f(c, \sigma)=p d f(\Psi(c, \sigma), \sigma) *|J|=
$$

$=\frac{1}{\sqrt{\pi t} \Psi(c, \sigma)} \frac{\lambda^{\theta}}{\Gamma(\theta)} \frac{1}{\sigma^{2(\theta+1)}} \exp \left(-\frac{\lambda}{\sigma^{2}}\right) \exp \left(-\frac{1}{4 \sigma^{2} t}\left[\ln \left(\frac{\Psi(c, \sigma)}{P_{0}}\right)-\left(m-\frac{1}{2} \sigma^{2}\right) t\right]^{2}\right) \frac{1}{\Phi\left(d_{1}^{*}\right)}$

where $d_{1}^{*}=\frac{\left[\ln \left(\frac{\Psi(c, \sigma)}{K e^{-r \tau}}\right)+\frac{\sigma^{2} \tau}{2}\right]}{\sigma \sqrt{\tau}}$.

Integrating out $\sigma$ will give us the prior density of the option price:

$$
p d f(c)=\int_{0}^{\infty} p d f(c, \sigma) \partial \sigma
$$

Note however that there is no closed form solution for this integral and it will have to be evaluated numerically. More of that in Section 3.

REMARK 2.3. We can utilise another procedure to obtain the marginal density of the option price $p d f(c)$. We start again from $p d f\left(P_{t}, \sigma\right)$ but this time we consider the transformation

$$
\begin{aligned}
c & =C\left(P_{t}, \sigma\right) \Rightarrow \Theta(c)=\Theta\left(c, P_{t}\right)=\sigma . \\
P_{t} & =P_{t}
\end{aligned}
$$

where $\Theta$ is the inverse of the option price with respect to $\sigma{ }^{13}$ This is commonly referred to as the implied volatility of the option price. The Jacobian $J$ of the transformation is given by

$$
\frac{1}{J}=\left|\begin{array}{ll}
\frac{\partial c}{\partial \sigma}=v e g a & \frac{\partial c}{\partial P_{t}}=\Phi\left(d_{1}\right) \\
\frac{\partial P_{t}}{\partial \sigma}=0 & \frac{\partial P_{t}}{\partial P_{t}}=1
\end{array}\right|=v e g a
$$

Thus we obtain pdf $\left(c, P_{t}\right)=p d f\left(\Theta\left(c, P_{t}\right), P_{t}\right) *|J|=$

$$
\frac{1}{\sqrt{\pi t} P_{t}} \frac{\lambda^{\theta}}{\Gamma(\theta)} \frac{1}{\Theta\left(c, P_{t}\right)^{2(\theta+1)}} \exp \left(-\frac{\lambda}{\Theta\left(c, P_{t}\right)^{2}}\right) \exp \left(-\frac{1}{4 \sigma^{2} t}\left[\ln \left(\frac{P_{t}}{P_{0}}\right)-\left(m-\frac{1}{2} \Theta\left(c, P_{t}\right)^{2}\right) t\right]^{2}\right) \frac{1}{v e g a}
$$

\footnotetext{
${ }^{13}$ We invert the option pricing formula in terms of $\sigma$, hence obtaining $\sigma$ as a function of $c$ and $P_{t}$. It should however be noted that there is no analytic expression (with the exception of an at-the-money option) for $\sigma=\Theta\left(P_{t}, c\right)$ and a Newton-Raphson numerical approximation is required.
} 
where vega $=\phi\left(\frac{\left[\ln \left(\frac{P_{t}}{K e^{-r \tau}}\right)+\frac{\Theta\left(c, P_{t}\right)^{2} \tau}{2}\right]}{\Theta\left(c, P_{t}\right) \sqrt{\tau}}\right) P_{t} \sqrt{\tau}$

Integrating out $P_{t}$ gives us the prior density of the option price: $p d f(c)=\int_{0}^{\infty} p d f\left(c, P_{t}\right) \partial P_{t}$.

\subsection{The posterior density}

In contrast to classical analysis where the main piece of output is a point estimate, Bayesian analysis produces as its main piece of output the so-called posterior density. This posterior density can then be combined with a loss or utility function to allow a decision to be made on the basis of minimising expected loss or maximising expected utility. For example, for positive definite quadratic loss functions the mean of the posterior distribution is an optimal point estimate. If the loss is proportional to the absolute value of the difference between the true and the estimated values, the median is chosen, while a zero loss for a correct estimate and a constant loss for an incorrect estimate leads to the choice of the mode.

To derive the posterior density of the option price, which unconditionally depends on two stochastic arguments (namely the price process and volatility) while conditionally only on volatility, we proceed as follows:

By Bayes rule

$$
p d f\left(P_{t}, \sigma \backslash s\right)=\frac{p d f\left(P_{t}, \sigma\right) p d f\left(s \backslash \sigma, P_{t}\right)}{p d f(s)}=\frac{p d f\left(P_{t}, \sigma\right) p d f(s \backslash \sigma)}{p d f(s)}=\frac{p d f\left(P_{t}, \sigma, s\right)}{p d f(s)}
$$

We therefore require expressions for $p d f\left(P_{t}, \sigma, s\right)$ and $p d f(s)$ :

PROPOSITION 2.2. $p d f\left(P_{t}, \sigma, s\right)=$

$\frac{2}{\sqrt{\pi t} P_{t}} \frac{\lambda^{\theta}}{\Gamma\left(\frac{v}{2}\right) \Gamma(\theta)}\left(\frac{v}{2}\right)^{\frac{v}{2}} s^{v-1} \frac{1}{\sigma^{2 \theta+v+2}} \exp \left(-\frac{2 \lambda+v s^{2}}{2 \sigma^{2}}\right) \exp \left(-\frac{1}{4 \sigma^{2} t}\left[\ln \left(\frac{P_{t}}{P_{0}}\right)-\left(m-\frac{1}{2} \sigma^{2}\right) t\right]^{2}\right)$

Proof. From Proposition 2.1 we have $p d f\left(P_{t}, \sigma\right)$. Also from Assumption 2.3 we have $p d f(s \backslash \sigma)\left[=p d f\left(s \backslash \sigma, P_{t}\right)\right]$. Then $p d f\left(P_{t}, \sigma, s\right)=p d f\left(P_{t}, \sigma\right) * p d f(s \backslash \sigma)$ and the result follows. 
PROPOSITION 2.3. The unconditional density of the statistic $s$ is given by:

$$
p d f(s)=\frac{2}{\mathrm{~B}\left(\frac{v}{2}, \theta\right)} \lambda^{\theta}\left(\frac{v}{2}\right)^{\frac{v}{2}} \frac{s^{v-1}}{\left(\lambda+\frac{v s^{2}}{2}\right)^{\frac{v}{2}+\theta}}
$$

Proof. From Assumptions 2.4 and 2.3 we know $p d f\left(\sigma^{2}\right)$ and $p d f\left(s^{2} \backslash \sigma^{2}\right)$. Then $p d f\left(s^{2}, \sigma^{2}\right)=p d f\left(\sigma^{2}\right)^{*} p d f\left(s^{2} \backslash \sigma^{2}\right)$. We can now integrate out $\sigma^{2}$ to obtain the marginal probability density of $s^{2}$ i.e. $\quad p d f\left(s^{2}\right)=\int_{0}^{\infty} p d f\left(s^{2}, \sigma^{2}\right) \partial \sigma^{2}$. Finally $p d f(s)=2 s^{*} p d f\left(s^{2}\right)$. The complete proof and all the relevant calculations are exhibited in Section A.2 in the Appendix.

Now take $p d f\left(P_{t}, \sigma, s\right)$ and consider the transformation:

$$
\begin{aligned}
& c=C(\sigma) \Leftrightarrow \sigma=C^{-1}(c)=\Theta(c) \\
& P_{t}=P_{t} \\
& s=s
\end{aligned}
$$

where $\Theta$ is the inverse of the option price with respect to $\sigma$. It should be noted that there is no analytic expression for $\sigma=\Theta(c)$ (with the exception of an at-the-money option, see Corollary 2.1 below) and a Newton-Raphson numerical approximation is required. The Jacobian of the transformation is given by:

$$
\frac{1}{J}=\left|\begin{array}{lll}
\frac{\partial c}{\partial \sigma}=v e g a & \frac{\partial c}{\partial P_{t}}=\Phi\left(d_{1}\right) & \frac{\partial c}{\partial s}=0 \\
\frac{\partial P_{t}}{\partial \sigma}=0 & \frac{\partial P_{t}}{\partial P_{t}}=1 & \frac{\partial P_{t}}{\partial s}=0 \\
\frac{\partial s}{\partial \sigma}=0 & \frac{\partial s}{\partial P_{t}}=0 & \frac{\partial s}{\partial s}=1
\end{array}\right|=\text { vega }
$$

Then

$$
p d f\left(P_{t}, c, s\right)=p d f\left(P_{t}, \Theta(c), s\right)^{*}|J| .
$$

We can now obtain an expression for $p d f\left(P_{t}, c \backslash s\right)$ : 


$$
p d f\left(P_{t}, c \backslash s\right)=\frac{p d f\left(P_{t}, c, s\right)}{p d f(s)}=
$$

$\frac{1}{\sqrt{\pi t} P_{t} \Gamma\left(\frac{v}{2}+\theta\right)} \frac{\left(\lambda+\frac{v s^{2}}{2}\right)^{\frac{v}{2}+\theta}}{\Theta(c)^{2 \theta+v+2}} \exp \left(-\frac{2 \lambda+v s^{2}}{2 \Theta(c)^{2}}\right) \exp \left(-\frac{1}{4 \Theta(c)^{2} t}\left[\ln \left(\frac{P_{t}}{P_{0}}\right)-\left(m-\frac{1}{2} \Theta(c)^{2}\right) t\right]^{2}\right) J \mid$

where $\quad J=1 /\left(\phi\left(\frac{\ln \left(\frac{P_{t}}{K e^{-r \tau}}\right)+\frac{\sigma^{2} \tau}{2}}{\sigma \sqrt{\tau}}\right) P_{t} \sqrt{\tau}\right)$.

Having obtained $p d f\left(P_{t}, c \backslash s\right)$; the posterior density of the option price is given by:

$$
p d f\left(c \backslash P_{t}, s\right)=\frac{p d f\left(P_{t}, c \backslash s\right)}{p d f\left(P_{t}\right)}
$$

We derived $p d f\left(P_{t}, c \backslash s\right)$ in (2.12), but we need an expression for $p d f\left(P_{t}\right)$.

PROPOSITION 2.4. The marginal density for the asset price is given by: $p d f\left(P_{t}\right)=$

$$
\frac{\lambda^{\theta} e^{-\frac{\ln \left(\frac{P_{t}}{P_{0}}\right)-m t}{4}}}{\sqrt{\pi t} P_{t} \Gamma(\theta)}\left(\frac{t}{16}\right)^{\theta+\frac{1}{2}}\left[\frac{4 \lambda t+\left(\ln \left(\frac{P_{t}}{P_{0}}\right)-m t\right)^{2}}{64}\right]^{-\frac{2 \theta+1}{4}} K_{\theta+\frac{1}{2}}\left(\frac{1}{4} \sqrt{4 \lambda t+\left(\ln \left(\frac{P_{t}}{P_{0}}\right)-m t\right)^{2}}\right)
$$

where $K_{\theta+\frac{1}{2}}\left(\frac{1}{4} \sqrt{4 \lambda t+\left(\ln \left(\frac{P_{t}}{P_{0}}\right)-m t\right)^{2}}\right)$ is the modified Bessel functionof the second

kind of order $\theta+\frac{1}{2} \cdot{ }^{14}$

${ }^{14}$ Modified Bessel functions are solutions to the differential equation $x^{2} y^{\prime \prime}+x y^{\prime}-\left(x^{2}+a^{2}\right) y=0$.

DEFINITION: Bessel functions

The differential equation $x^{2} y^{\prime \prime}+x y^{\prime}+\left(x^{2}-a^{2}\right) y=0$ is known as the Bessel equation where $a$ is a non-negative constant. Some of its solutions are known as Bessel functions. The function $J_{a}$ defined by $J_{a}(x)=\sum_{n=0}^{\infty} \frac{(-1)^{n}}{n !(n+a) !}\left(\frac{x}{2}\right)^{2 n+a}$ for $x>0$ and $a$ a nonnegative integer is called the Bessel function of the first kind of order $a$. The function $K_{a}$ defined for $x>0$ by 
Proof. In Proposition 2.1 we have obtained $p d f\left(P_{t}, \sigma\right)$, which we can straightforwardly transform to $p d f\left(P_{t}, \sigma^{2}\right)$. Then the result follows from the fact that $p d f\left(P_{t}\right)=\int_{0}^{\infty} p d f\left(P_{t}, \sigma^{2}\right) \partial \sigma^{2}$ can be written as

$\mathrm{A}^{*} \int_{0}^{\infty} \frac{c^{\omega}}{\Gamma(\omega)}\left(\frac{1}{\sigma^{2}}\right)^{\omega+1} \exp \left(-\frac{c}{\sigma^{2}}\right) \exp \left(-p \sigma^{2}\right) \partial \sigma^{2}$

where $\mathrm{A}$ is a constant. It is easy now to observe that the integral is the Laplace transform of an Inverted-Gamma function:

$L\left(f_{i \gamma}\right)=F(p)=\int_{0}^{\infty} f_{i \gamma}\left(\sigma^{2}\right) e^{-p \sigma^{2}} d \sigma^{2}$.

For the complete proof see section A.3. in the Appendix.

We have now completed the derivation of the posterior density of the BS option price. Let us present here the full expression:

$$
p d f\left(c \backslash P_{t}, s\right)=\frac{p d f\left(P_{t}, c \backslash s\right)}{p d f\left(P_{t}\right)}=
$$

$$
=\frac{\Gamma(\theta)\left(\lambda+\frac{v s^{2}}{2}\right)^{\frac{v}{2}+\theta} \exp \left(-\frac{2 \lambda+v s^{2}}{2 \Theta(c)^{2}}-\frac{1}{4 \Theta(c)^{2} t}\left[\ln \left(\frac{P_{t}}{P_{0}}\right)-\left(m-\frac{1}{2} \Theta(c)^{2}\right) t\right]^{2}+\frac{\ln \left(\frac{P_{t}}{P_{0}}\right)-m t}{4}\right) *\left|J^{\prime}\right|}{\Gamma\left(\frac{v}{2}+\theta\right) \lambda^{\theta} \Theta(c)^{2 \theta+v+2}\left(\frac{t}{16}\right)^{\theta+\frac{1}{2}}\left(\frac{4 \lambda t+\left(\ln \left(\frac{P_{t}}{P_{0}}\right)-m t\right)^{2}}{64}\right)^{-\frac{2 \theta+1}{4}} K_{\theta+\frac{1}{2}}\left(\frac{1}{4} \sqrt{4 \lambda t+\left(\ln \left(\frac{P_{t}}{P_{0}}\right)-m t\right)^{2}}\right)}
$$

where $J^{\prime}=1 /\left(\phi\left(\frac{\ln \left(\frac{P_{t}}{K e^{-r \tau}}\right)+\frac{\Theta(c)^{2} \tau}{2}}{\Theta(c) \sqrt{\tau}}\right) P_{t} \sqrt{\tau}\right)$

$K_{a}(x)=J_{a}(x) \ln x-\frac{1}{2}\left(\frac{x}{2}\right)^{-a} \sum_{n=0}^{a-1} \frac{(a-n-1) !}{n !}\left(\frac{x}{2}\right)^{2 n}-\frac{1}{2}\left(\frac{x}{2}\right)^{a} \sum_{n=0}^{\infty}(-1)^{n} \frac{h_{n}+h_{n+a}}{n !(n+a) !}\left(\frac{x}{2}\right)^{2 n}$

is called the Bessel function of the second kind of order $a$. The general solution of the Bessel equation in this case for $x>0$ is $y=c_{1} J_{a}(x)+c_{2} K_{a}(x)$.

For a simple exposition of Bessel functions see for example Apostol (1969, Volume II, pp. 182-190). 
COROLLARY 2.1. If the option is at-the-money, i.e. $P_{t}=K \exp (-r \tau)$, then certain simplifications occur: $\Theta(c)=\frac{2}{\sqrt{\tau}} \Phi^{-1}\left(\frac{1}{2}\left(\frac{c}{P_{t}}+1\right)\right)$ and $J^{\prime}=1 /\left(\phi\left(\Phi^{-1}\left(\frac{1}{2}\left(\frac{c}{P_{t}}+1\right)\right)\right) P_{t} \sqrt{\tau}\right)$. $\Phi^{-1}(. .$.$) denotes the inverse cumulative normal distribution function.$

Proof. When the option is at-the-money, the BS formula (given in equation (2.5)) simplifies to $c=C\left(P_{t}=K \exp (-r \tau), \sigma\right)=P_{t}\left(\Phi\left(\frac{\sigma \sqrt{\tau}}{2}\right)-\Phi\left(-\frac{\sigma \sqrt{\tau}}{2}\right)\right)=P_{t}\left(2 \Phi\left(\frac{\sigma \sqrt{\tau}}{2}\right)-1\right)$. This then implies that: $\Phi\left(\frac{\sigma \sqrt{\tau}}{2}\right)=\frac{1}{2}\left(\frac{c}{P_{t}}+1\right) \Rightarrow \sigma=\Theta(c)=\frac{2}{\sqrt{\tau}} \Phi^{-1}\left(\frac{1}{2}\left(\frac{c}{P_{t}}+1\right)\right)$. Also for $P_{t}=K \exp (-r \tau)$ we have $J^{\prime}=1 /\left(\phi\left(\frac{\Theta(c) \sqrt{\tau}}{2}\right) P_{t} \sqrt{\tau}\right)$. Substituting in, the analytic expression for $\Theta(c)$ we get the proposed result for $J^{\prime}$.

REMARK 2.4. The at-the-money case is best interpreted as a stochastic exercise price where $K=P_{t} \exp (r \tau)$.

REMARK 2.5. It is interesting to observe that the posterior density of the option price does depend on the expected rate of return $\mu$ through the hyperparameter $m$ ( $m$ represents our prior beliefs about $\mu$ ). The true unknown $\mu$ has been integrated out. The existence of $m$ in the formula is due to randomness in prices prior to sampling.

Having derived the prior and posterior densities of the BS option price (i.e. $p d f(c)$ and $\left.p d f\left(c \backslash P_{t}, s\right)\right)$ it is interesting, for comparative purposes in particular, to derive expressions for $p d f(c \backslash s)$ and $p d f\left(c \backslash P_{t}\right)$. This way we can illustrate how the dispersion of the density of the option price changes as we condition on more information: from the prior $p d f(c)$, to conditioning only on the sample estimate of volatility $p d f(c \backslash s)$, to conditioning on the price $p d f\left(c \backslash P_{t}\right)$, to the posterior density $p d f\left(c \backslash P_{t}, s\right)$. We refer the reader to Section A.4. in the Appendix for the derivation of $p d f(c \backslash s)$ and $p d f\left(c \backslash P_{t}\right)$.

It should be stressed that randomness in prices and volatility has been assumed throughout our analysis. We write $c=C\left(P_{t}, \sigma\right)$ to denote that fact. For fixed prices but random volatility we would write $c=C(\sigma)$. Note for example that $p d f\left(c=C\left(P_{t}, \sigma\right) \backslash s\right)$ and $p d f(c=C(\sigma) \backslash s)$ represent two very different densities. ${ }^{15}$

\footnotetext{
${ }^{15}$ The former represents the density of the option price conditional on the sample estimate of volatility but with prices unknown, while the latter represents the density of the option price conditional on the sample estimate of volatility but with prices known and fixed.
} 
REMARK 2.6. So far it has been assumed that $c=C\left(P_{t}, \sigma\right)$. Karolyi (1993) assumes that prices are non-random (i.e. $c=C(\sigma)$ ) and suggests that the posterior density of the option price can be derived as a non-linear transformation of the posterior density of volatility. Let us briefly illustrate how $p d f(c=C(\sigma) \backslash s)$ can be obtained:

The posterior density of volatility is given by

$$
p d f(\sigma \backslash s)=\frac{p d f(\sigma)^{*} L(\sigma \backslash s)}{\int_{0}^{\infty} p d f(\sigma, s) \partial \sigma}=\frac{p d f(\sigma, s)}{p d f(s)}
$$

where

$$
\begin{aligned}
p d f(\sigma, s) & =p d f(\sigma)^{*} p d f(s \backslash \sigma) \\
& =\frac{4 \lambda^{\theta}}{\Gamma(\theta) \Gamma(v / 2)}\left(\frac{v}{2}\right)^{v / 2} \frac{s^{v-1}}{\sigma^{2 \theta+v+1}} \exp \left(-\frac{2 \lambda+v s^{2}}{2 \sigma^{2}}\right)
\end{aligned}
$$

Also from Proposition 2.3 we have:

$$
p d f(s)=\frac{2}{\mathrm{~B}\left(\frac{v}{2}, \theta\right)} \lambda^{\theta}\left(\frac{v}{2}\right)^{\frac{v}{2}} \frac{s^{v-1}}{\left(\lambda+\frac{v s^{2}}{2}\right)^{\frac{v}{2}+\theta}}
$$

Then

$$
p d f(\sigma \backslash s)=\frac{2}{\Gamma\left(\frac{v}{2}+\theta\right)} \frac{\left(\lambda+\frac{v s^{2}}{2}\right)^{\frac{v}{2}+\theta}}{\sigma^{2 \theta+v+1}} \exp \left(-\frac{2 \lambda+v s^{2}}{2 \sigma^{2}}\right)
$$

This is the posterior density of $\sigma$. Using the transformation $c=C(\sigma)$, i.e. inverting the Black-Scholes formula in terms of $\sigma=C^{-1}(c)=\Theta(c)$ we obtain $p d f(c=C(\sigma) \backslash s)$ :

$$
p d f(c=C(\sigma) \backslash s)=\frac{2\left(\lambda+\frac{v s^{2}}{2}\right)^{\frac{v}{2}+\theta} \exp \left(-\frac{2 \lambda+v s^{2}}{2[\Theta(c)]^{2}}\right)}{\Gamma\left(\frac{v}{2}+\theta\right)[\Theta(c)]^{2 \theta+v+1} \phi\left(\frac{\ln \left(\frac{P_{t}}{K e^{-r \tau}}\right)+\frac{[\Theta(c)]^{2} \tau}{2}}{\Theta(c) \sqrt{\tau}}\right) P_{t} \sqrt{\tau}}
$$

Again, for the at-the-money case, the simplifications outlined in Corollary 2.1 apply. 


\section{Numerical Evaluation}

In Equation (2.7) we have derived the joint unconditional density of the option price and volatility:

$$
\begin{aligned}
& p d f(c, \sigma)= \\
& \frac{1}{\sqrt{\pi t} \Psi(c, \sigma)} \frac{\lambda^{\theta}}{\Gamma(\theta)} \frac{1}{\sigma^{2 \theta+2}} \exp \left(-\frac{\lambda}{\sigma^{2}}\right) \exp \left(-\frac{1}{4 \sigma^{2} t}\left[\ln \left(\frac{\Psi(c, \sigma)}{P_{0}}\right)-\left(m^{\prime}-\frac{1}{2} \sigma^{2}\right) t\right]^{2}\right) * \\
& * \frac{1}{\Phi\left[\frac{\ln (\Psi(c, \sigma) / K)+\left(r+1 / 2 \sigma^{2}\right) \tau}{\sigma \sqrt{\tau}}\right]}
\end{aligned}
$$

where

$\Psi(c, \sigma)$ is the inverse of the option price $c$ with respect to $P_{t}$. Call it the implied price hereafter.

$\lambda, \theta$ are the prior parameters of the volatility distribution.

$m$ is the prior expected rate of return of the asset.

$t$ is the sample size (it is reasonable to assume that the sample size is known although the sample is not yet drawn).

$\tau$ is the time to maturity of the option under consideration.

$P_{0}, K$, and $r$ are the initial asset price, the strike price, and the risk free interest rate respectively.

$\Phi($.$) is the cumulative standard normal distribution function.$

To find the marginal (prior) density of the option price we need to integrate out the volatility parameter $\sigma$. However, this cannot be done analytically and we will have to evaluate the density numerically.

Let us first specify our prior parameters; namely $\lambda, \theta$ and $m$. We have from Assumption 2.4 that:

$$
p d f(\sigma)=2 \sigma \frac{\lambda^{\theta}}{\Gamma(\theta)} \frac{1}{\left(\sigma^{2}\right)^{\theta+1}} \exp \left(-\frac{\lambda}{\sigma^{2}}\right)
$$

Taking the first and second moments of the distribution of volatility we get

$$
E(\sigma)=\int_{0}^{\infty} \sigma[\underbrace{\left[2 \sigma \frac{\lambda^{\theta}}{\Gamma(\theta)} \frac{1}{\left(\sigma^{2}\right)^{\theta+1}} \exp \left(-\frac{\lambda}{\sigma^{2}}\right)\right.}_{p d f(\sigma \backslash \lambda, \theta)}] d \sigma
$$




$$
=\lambda^{1 / 2} \frac{\Gamma(\theta-1 / 2)}{\Gamma(\theta)} \underbrace{\int_{0}^{\infty} 2 \sigma \frac{\lambda^{\theta-1 / 2}}{\Gamma(\theta-1 / 2)} \frac{1}{\left(\sigma^{2}\right)^{\left(\theta-\frac{1}{2}\right)+1}} \exp \left(-\frac{\lambda}{\sigma^{2}}\right) d \sigma}_{=1}
$$

Hence

$$
E(\sigma)=\lambda^{1 / 2} \frac{\Gamma(\theta-1 / 2)}{\Gamma(\theta)}
$$

Also

$$
\begin{aligned}
E\left(\sigma^{2}\right) & =\int_{0}^{\infty} \sigma^{2}\left[2 \sigma \frac{\lambda^{\theta}}{\Gamma(\theta)} \frac{1}{\left(\sigma^{2}\right)^{\theta+1}} \exp \left(-\frac{\lambda}{\sigma^{2}}\right)\right] d \sigma \\
& =\frac{\lambda}{\theta-1} \int_{0}^{\infty} 2 \sigma \frac{\lambda^{\theta-1}}{\Gamma(\theta-1)} \frac{1}{\left(\sigma^{2}\right)^{(\theta-1)+1}} \exp \left(-\frac{\lambda}{\sigma^{2}}\right) d \sigma
\end{aligned}
$$

Thus

$$
E\left(\sigma^{2}\right)=\frac{\lambda}{\theta-1}
$$

And

$$
\operatorname{Var}(\sigma)=E\left(\sigma^{2}\right)-[E(\sigma)]^{2} \quad \Rightarrow \quad E\left(\sigma^{2}\right)=\operatorname{Var}(\sigma)+[E(\sigma)]^{2}
$$

Once we have prior beliefs about the mean and variance of volatility (i.e. $E(\sigma)$ and $\operatorname{Var}(\sigma))$ we can calculate $\lambda$ and $\theta$ using equations (3.1), (3.2), and (3.3) above. Our prior beliefs, i.e. $E(\mu)$, will also determine the value of $m$. We digress briefly to discuss how $\lambda$ and $\theta$ might be chosen.

One version is the "empirical" Bayes approach. A prior is constructed from the data themselves, and so can be viewed as incorporating a non-informative prior. The Stein estimator, can be viewed as an empirical Bayes estimator. (see Efron and Morris, (1973)).

Prior sample data could also act as a useful source of information when forming prior beliefs. This is because of the clustering effect: observations of financial time series reveal bunching of high and low volatility episodes. Alternatively, one could use the long run average of volatility as prior information to capture the mean reverting behaviour of volatility. ${ }^{16}$

We want to solve the Black-Scholes equation in terms of $P_{t}$ and thus obtain $P_{t}=\Psi(c, \sigma)$ (i.e. obtain the price of the underlying as a function of the option price and of volatility). But

$$
c=P_{t} \Phi\left(\frac{\log \left[\frac{P_{t}}{K}\right]+\left[r+\frac{1}{2} \sigma^{2}\right] \tau}{\sigma \sqrt{\tau}}\right)-K \exp (-r \tau) \Phi\left(\frac{\log \left[\frac{P_{t}}{K}\right]+\left[r-\frac{1}{2} \sigma^{2}\right] \tau}{\sigma \sqrt{\tau}}\right)
$$

\footnotetext{
${ }^{16}$ For stylised facts in volatility see for example Ghysels, Harvey, and Renault (1996).
} 
cannot be inverted in closed form in terms of $P_{t}$ (with the exception of an at-themoney option). Instead we will calculate numerical values for $\Psi(c, \sigma)$. We evaluate $\Psi\left(c_{i}, \sigma_{j}\right)$ for $i=1, \ldots, n$ and $j=1, \ldots, u$ spanning (with the desired degree of accuracy) all possible values of $c$ and $\sigma$, thus generating an $n \times u$ matrix of implied prices:

$$
\Psi(c, \sigma)=\left[\begin{array}{cccc}
\Psi\left(c_{1}, \sigma_{1}\right) & \Psi\left(c_{1}, \sigma_{2}\right) & \ldots & \Psi\left(c_{1}, \sigma_{u}\right) \\
\Psi\left(c_{2}, \sigma_{1}\right) & \Psi\left(c_{2}, \sigma_{2}\right) & \ldots & \ldots \\
\ldots & \ldots & . . & \ldots \\
\Psi\left(c_{n}, \sigma_{1}\right) & \ldots & \ldots & \Psi\left(c_{n}, \sigma_{u}\right)
\end{array}\right]
$$

To ensure conformability in the calculations to follow we also generate an $n \times u$ matrix for $\sigma$ of the form:

$$
\sigma=\left[\begin{array}{ccc}
\sigma_{1} & \ldots & \sigma_{u} \\
\ldots & \ldots & \ldots \\
\sigma_{1} & \ldots & \sigma_{u}
\end{array}\right]
$$

Substituting $\Psi(c, \sigma)$ and $\sigma$ in the formula for the joint density (2.7), we obtain an $n$ $x u$ matrix of values for $p d f(c, \sigma)$.

$$
p d f(c, \sigma)=\left[\begin{array}{ccc}
p d f\left(c_{1}, \sigma_{1}\right) & \ldots & p d f\left(c_{1}, \sigma_{u}\right) \\
\ldots & \ldots & \ldots \\
p d f\left(c_{n}, \sigma_{1}\right) & \ldots & p d f\left(c_{n}, \sigma_{u}\right)
\end{array}\right]
$$

It should be noted that all the products between matrices that occur in the calculation of $p d f(c, \sigma)$ are Hadamard (elementwise) products.

It is now straightforward to obtain $p d f(c)$ :

$$
p d f(c) \approx \sum_{j=1}^{u} p d f\left(c_{i}, \sigma_{j}\right) \Delta j
$$

Turning to the posterior density $p d f\left(c \backslash P_{t}, s\right)$ (given in Equation (2.15)), we need to evaluate $\Theta(c)$. Remember $\Theta(c)$ is the inverse of the option price $c$ with respect to $\sigma$. Call it the implied volatility hereafter. However the BS formula cannot be inverted in closed form in terms of $\sigma$ (with the exception of an at-the-money option). Instead we calculate numerical values for $\Theta(c)$. We evaluate $\Theta\left(c_{i}\right)$ for $i=1, \ldots, n$ spanning with the desired degree of accuracy the range of values of $c$, thus generating an $n$ dimensional vector of implied volatilities. 


\section{Results}

Typical values of the volatility of a stock are in the range of $20 \%$ to $40 \%$ per annum. Assuming that time is measured in trading days and that there are 252 trading days per year we have calculated (from prior 30-day data) that the expected rate of return of the FTSE 100 index ${ }^{17}$ is $15 \%$ per annum. We have also calculated that the volatility is $25 \%$ per annum. The standard error of our estimate is approximately $\frac{0.25}{\sqrt{2 * 30}}=3.2 \%$ per annum.

Thus at time 0 , our "prior" information is that:

$E(\mu)=m=15 \%$ per annum or 0.0006 daily.

$E(\sigma)=25 \%$ per annum, or 0.0158 daily.

Std. Dev. $(\sigma)=3.2 \%$ per annum, or 0.002 daily.

$\operatorname{Var}(\sigma)=0.001024$ per annum, or $4^{-06}$ daily.

Also using equation (3.3) we calculate

$E\left(\sigma^{2}\right)=0.063524$ per annum, or $2.5364^{-04}$ daily.

Since we know $E(\sigma)$ and $E\left(\sigma^{2}\right)$ we can now calculate values for the prior parameters $\lambda$, and $\theta$ using equations (3.1) and (3.2). To sum up, our "prior beliefs" (in daily format) are: $\lambda=0.004, \theta=16.72$, and $m=0.0006$. Also the value of the index at time 0 is $P_{0}=2200$.

Turning to the sample information, we have that at time $t=30$ : The value of the index is $P_{t}=2206$. The daily sample standard deviation is $s=0.016$, and $v=t-1=$ 29. Our data are chosen to conform with values presented in Ncube and Satchell (1997).

Market information: Consider now a time $t$ European Call option on the FTSE 100 index with exercise prices $K=2025, K=2225$, and $K=2425, \tau=15$ (i.e. 15 trading days to maturity). The risk-free rate is $\mathrm{r}=0.0002$ (daily).

In Figure 4.1 we plot the prior density of the option price and the density of the option price conditional on the sample estimate of volatility for the case $K=2025$. (Note that the latter density is for illustrative purposes, rather than of any practical use or theoretical significance).

\footnotetext{
${ }^{17}$ The FTSE 100 index is an index for shares of stock of the top 100 companies that are traded in the London Stock Exchange. Options written on the index are traded in the London International Financial Futures Exchange.
} 


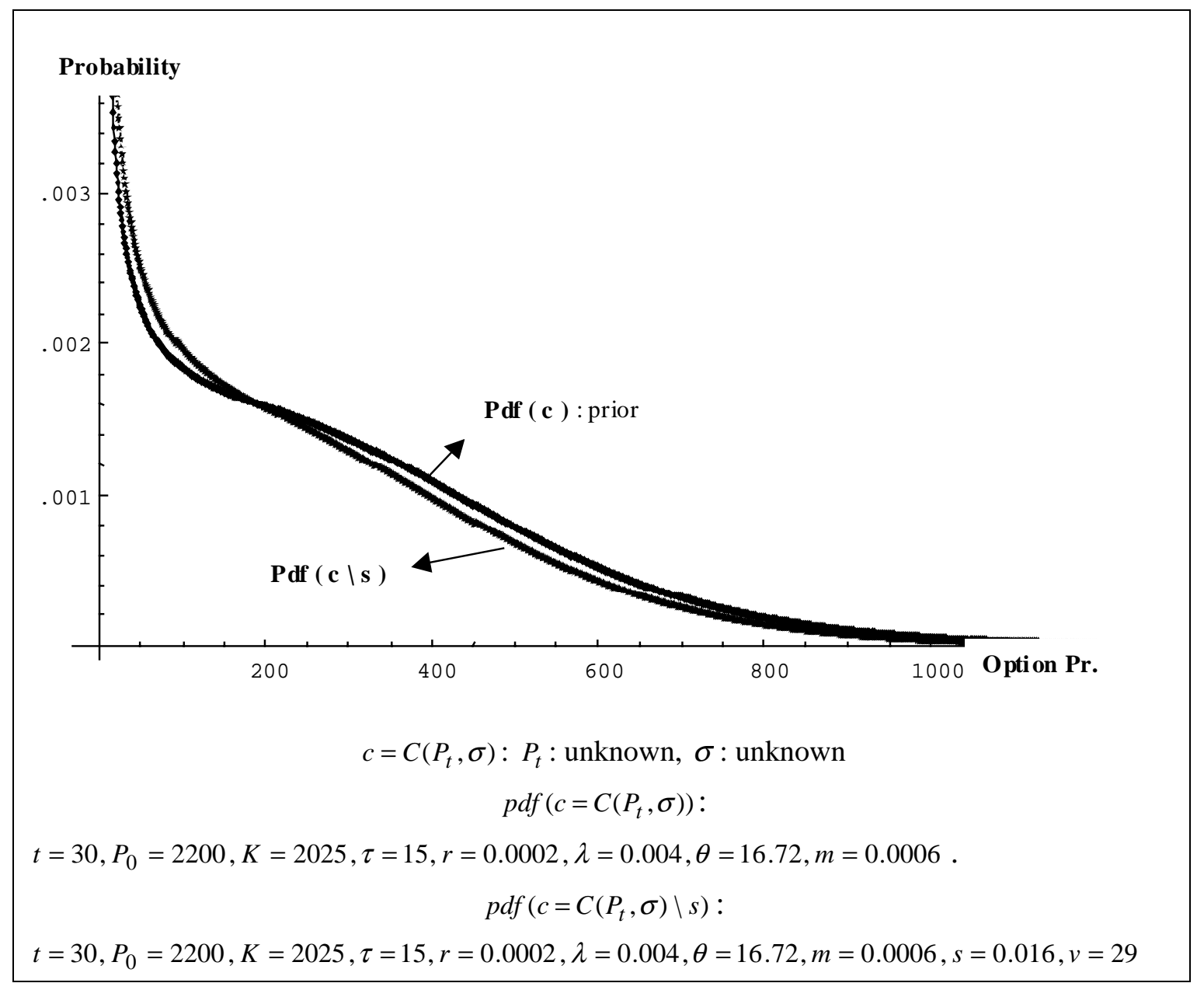

Figure 4.1. Prior $(p d f(c))$, and conditional on the sample estimate of volatility $(p d f(c \backslash s)$ ) probability density functions for the BS option price.

Observe that not conditioning on the asset price induces a very large dispersion in the option price. This effect is magnified since we are looking ahead 30 trading days $(t=30)$. Note also that conditioning on the sample estimate of volatility, when the underlying price is unknown, does not offer much improvement in reducing the dispersion of the option price.

In Table 4.1 we report $2.5 \%$ and $97.5 \%$ quantiles for the densities exhibited in Figure 4.1. To illustrate the effect of how the dispersion of the option price decreases as the conditioning horizon decreases, we also report quantiles for the cases $t=25,20$, 15,10 , and 5. A graphical illustration of the prior density of the BS option price for differing values of $t$ is exhibited in Figure 4.2.

At the bottom of Table 4.1 we also report summary statistics for the distribution of the underlying (i.e. the log-normal distribution) for $P_{0}=2200, t=30$ and for $\mu=m=0.0006$ and $\sigma=s=0.016$. To this end, we report the values of the option price that correspond to the $2.5 \%$ and $97.5 \%$ quantiles of the distribution of the asset price. Note that in order to calculate BS prices, we assume that volatility is known and equal to its sample estimate (i.e. $\sigma=s=0.016$ ). 
Table 4.1

2.5\% and 97.5\% quantiles of $p d f(c)$ and $p d f(c \backslash s)$ for $t=30,25,20,15,10$, and 5 .

\begin{tabular}{cccc}
\hline$p d f(c):$ Prior & 0.025 & 0.975 & Mean of $p d f(c)$ \\
\hline$t=30$ & 0.1 & 828.9 & 262.9 \\
25 & 0.4 & 751.7 & 252.2 \\
20 & 1.7 & 679.1 & 240.4 \\
15 & 5.2 & 606.5 & 227.6 \\
10 & 14.9 & 523.4 & 214.1 \\
5 & 39.8 & 416.2 & 200.2 \\
\hline$p d f(c \backslash s)$ & 0.025 & 0.975 & Mean of $p d f(c \backslash s)$ \\
\hline$t=30$ & 0.1 & 786.9 & 234.1 \\
25 & 0.1 & 712.0 & 227.6 \\
20 & 0.8 & 646.2 & 220.3 \\
15 & 3.6 & 583.0 & 212.4 \\
10 & 11.5 & 509.1 & 204.1 \\
5 & 37.2 & 410.4 & 195.6 \\
\hline
\end{tabular}

Summary statistics for the lognormal distribution: $P_{0}=2200, t=30, \mu=0.0006, \sigma=0.016$ and BS prices for $P_{t}=1879.2$ and $P_{t}=2649.5,(K=2025, \tau=15, \sigma=0.016, r=0.0002)$.

\begin{tabular}{lcccccc}
\hline & 0.025 & 0.975 & Mean & Std. Dev. & Skewness & Exs. Kurtosis \\
\hline$p d f\left(P_{t} \backslash P_{0}, \mu, \sigma\right):$ & 1879.2 & 2649.5 & 2240 & 196.7 & 0.264 & 0.124 \\
\hline BS price: $\quad \mathbf{7 . 3 8}$ & $\mathbf{6 3 0 . 5 4}$ & & & & & \\
\hline
\end{tabular}

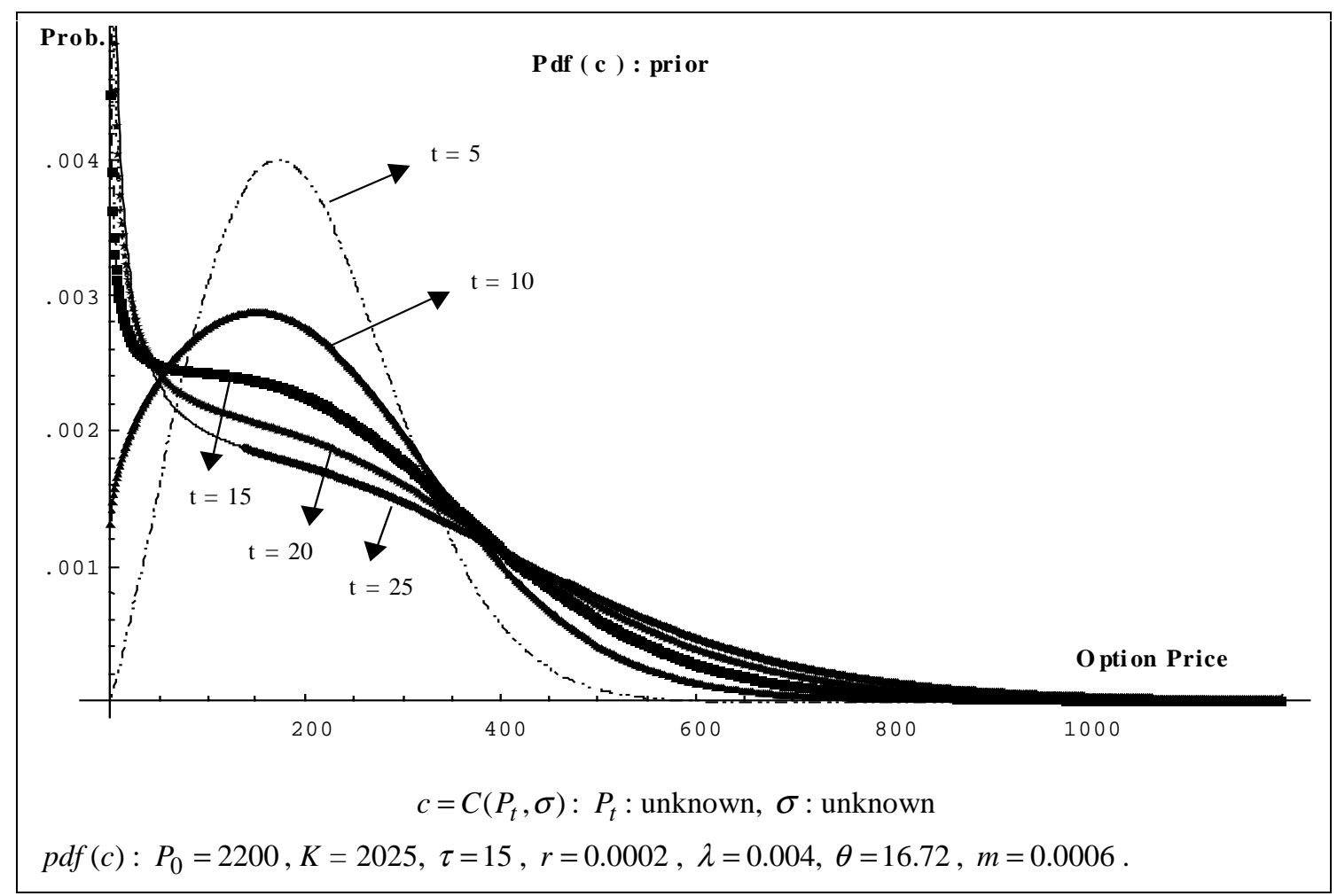

Figure 4.2. Prior densities for the BS option price for differing values of $t$. 
From Table 4.1, it is interesting to observe that (once we condition on $s$ ) the true 95\% range of the option price, given by $p d f(c \backslash s)$, is wider than the one we would obtain if we took advantage of the monotonicity properties of the BS option price with respect to the underlying, and used the lognormal distribution to derive $95 \%$ confidence intervals for the option price. To do the latter, one has to assume that randomness arises from the asset price while volatility is known and equal to its sample estimate. This is the approach of Ncube and Satchell (1997). If we do not condition on $s$, the true $95 \%$ range of the option price, given by $p d f(c)$, is even wider.

We now turn to the posterior density where we condition on the asset price $P_{t}$ and on the sample estimate of volatility $s$. Let us first illustrate the effect of varying $t$ values for the posterior density. In Figure 4.3 we plot the posterior density of the BS option price for $t=30,20,10,5$ and $K=2025, P_{t}=2206$, and $s=0.016$ (everything else as already defined above).

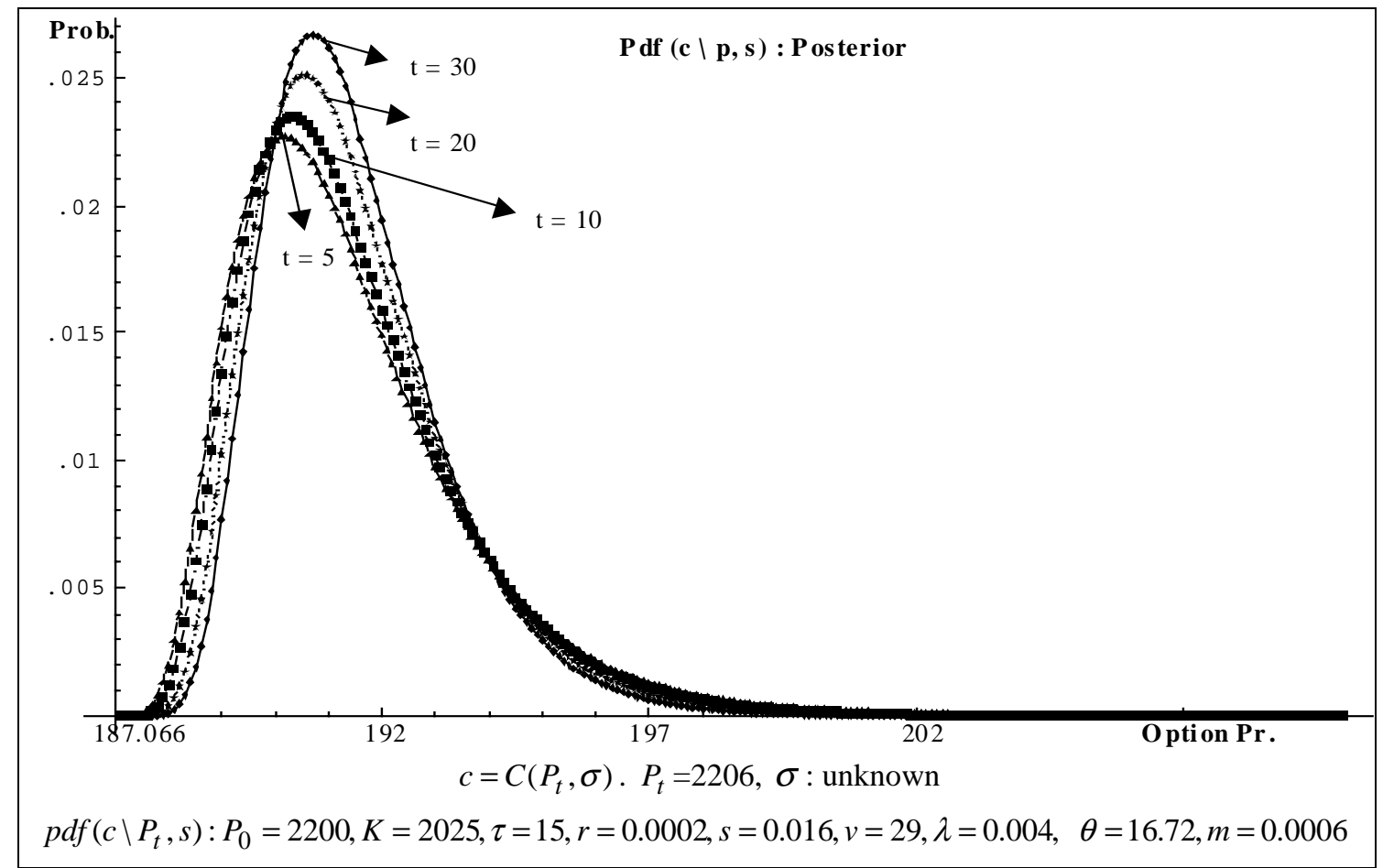

Figure 4.3. Posterior densities of the BS option price for differing values of $t$.

This time we observe the opposite effect of what we saw for the prior density. That is, the larger the sample size $t$ the smaller the dispersion in the option price (see Figure 4.3 and Table 4.2).

Table 4.2

$2.5 \%$ and $97.5 \%$ quantiles of $p d f\left(c \backslash P_{t}, s\right)$ for $t=30,20,10$, and 5 .

\begin{tabular}{|c|c|c|c|}
\hline Quantiles & 0.025 & 0.975 & Mean of $p d f\left(c \backslash P_{t}, s\right)$ \\
\hline$t=30$ & 188.9 & 195.2 & 189.8 \\
\hline 20 & 188.7 & 196.4 & 189.8 \\
\hline 10 & 188.5 & 196.9 & 190.1 \\
\hline 5 & 188.3 & 197.2 & 190.4 \\
\hline
\end{tabular}


Indeed a large sample size will provide a better estimate for the volatility (provided that the sample size is not too large, to avoid issues of non-stationarity) and hence reduce estimation risk. Despite the fact that for a large $t$ the prior density will be less informative (see Figure 4.2), the sample information is more robust and this is reflected in the posterior density

Comparing Figures 4.1 and 4.2, with Figure 4.3 it is obvious that conditioning on the asset price dramatically reduces the variability of the option price. We now present graphs to illustrate how the dispersion of the option price changes from conditioning on the asset price only, to conditioning on both the asset price and the sample estimate of volatility. In other words, we compare the density of the option price conditional on the asset price (i.e. $\left.p d f\left(c \backslash P_{t}\right)\right)$ with the posterior density (i.e. $\left.p d f\left(c \backslash P_{t}, s\right)\right)$. We do this for an in-the-money-option (i.e. $K=2025$ and $P_{t}=2206$ ), a near-the-money option (i.e. $K=2225$ and $P_{t}=2206$ ), an at-the-money option (i.e. $K=2212.63$ and $P_{t}=2206$ ), and an out-of-the-money option (i.e. $K=2425$ and $\left.P_{t}=2206\right)$. We present our results, complete with summary statistics for each distribution in Figures 4.4, 4.5, and 4.6 and Tables 4.3, 4.4, and 4.5 respectively.

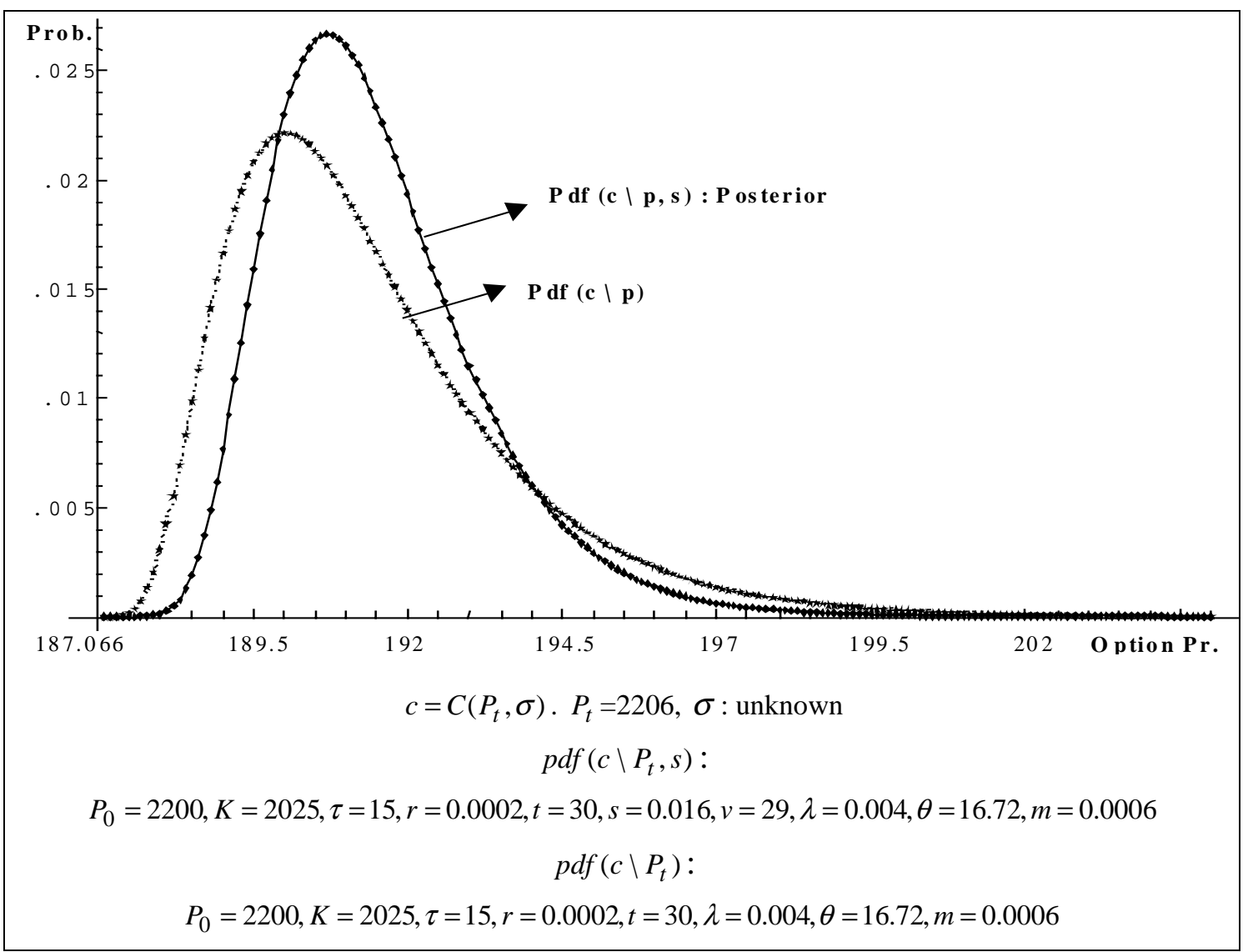

Figure 4.4. Posterior, and conditional (on the asset price) probability density functions for the BS option price. In-the-money case. 
Table 4.3

Summary statistics of distributions exhibited in Figure 4.4

\begin{tabular}{lcccccc}
\hline Quantiles & 0.025 & 0.975 & Mean $^{18}$ & Std. Dev. & Skewness & Exs. Kurtosis \\
\hline$p d f\left(c \backslash P_{t}, s\right):$ & 188.9 & 195.2 & 189.8 & 2.3 & 1.81 & 1.12 \\
\hline$p d f\left(c \backslash P_{t}\right):$ & 188.3 & 197.2 & 191.2 & 2.3 & 1.52 & 3.00 \\
\hline
\end{tabular}

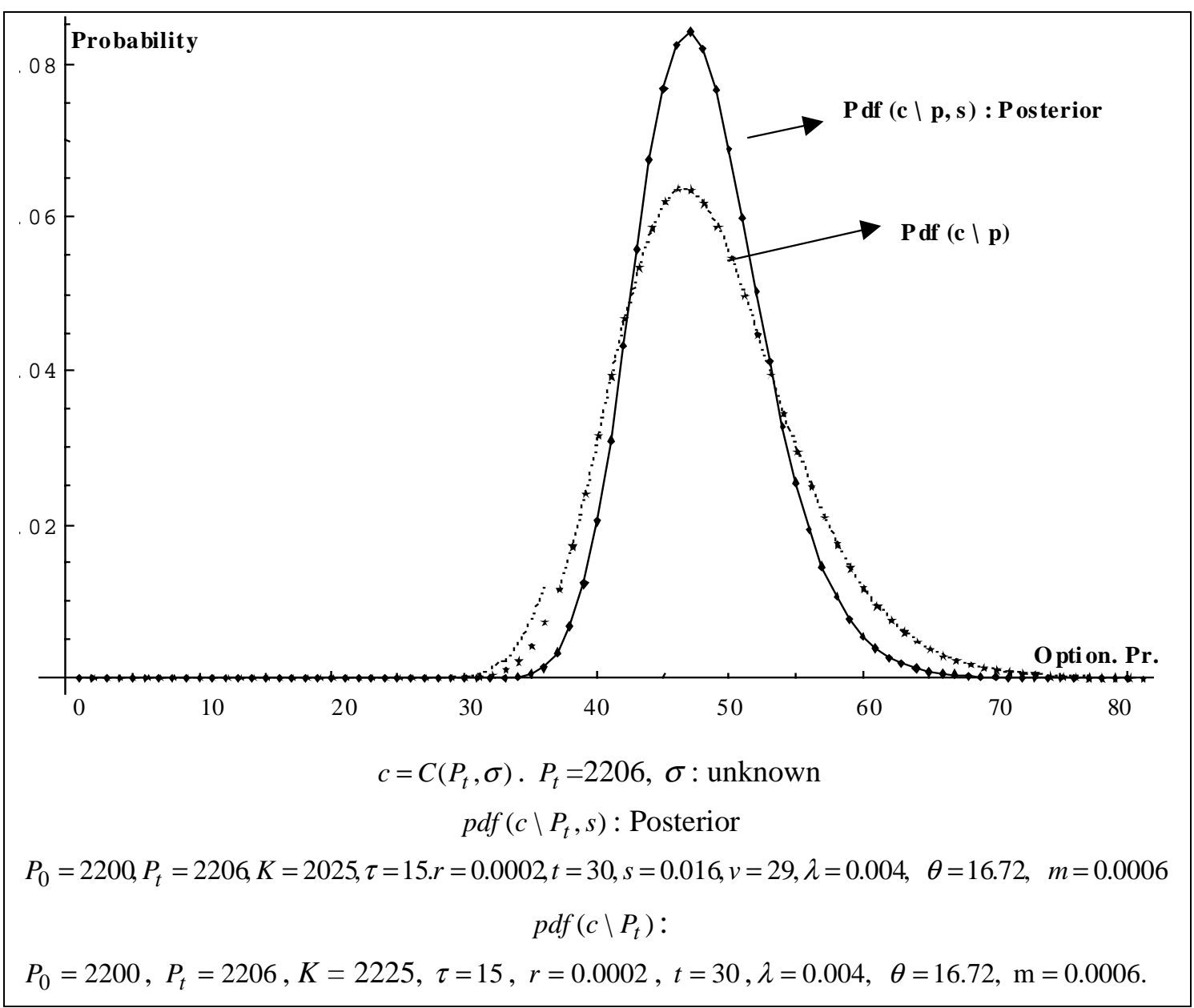

Figure 4.5. Posterior, and conditional (on the asset price) probability density functions for the BS option price. Near-the-money case.

Table 4.4.

Summary statistics of distributions exhibited in Figure 4.5 and at-the-money case.

Near-the-money (Figure 4.5)

\begin{tabular}{lcccccc}
\hline Quantiles & 0.025 & 0.975 & Mean & Std. Dev. & Skewness & Exs. Kurtosis \\
\hline$p d f\left(c \backslash P_{t}, s\right):$ & 39.0 & 59.1 & 47.6 & 4.85 & 0.70 & 0.57 \\
\hline$p d f\left(c \backslash P_{t}\right):$ & 35.8 & 61.6 & 47.3 & 6.60 & 0.62 & 0.64 \\
\hline
\end{tabular}

\footnotetext{
${ }^{18}$ If we combine the posterior density with a quadratic loss function, the mean of the posterior distribution is an optimal point estimate.
} 
At-the-money: $P_{t}=K \exp (-r \tau) .(K=2212.63$, everything else as in Figure 4.5)

\begin{tabular}{lrrrccc}
\hline \multicolumn{1}{c}{ Quantiles } & 0.025 & 0.975 & Mean & Std. Dev. & Skewness & Exs. Kurtosis \\
\hline$p d f\left(c \backslash P_{t}, s\right):$ & 44.7 & 64.9 & 53.3 & 4.86 & 0.74 & 0.61 \\
\hline$p d f\left(c \backslash P_{t}\right):$ & 41.4 & 73.9 & 53.1 & 6.62 & 0.64 & 0.78 \\
\hline
\end{tabular}

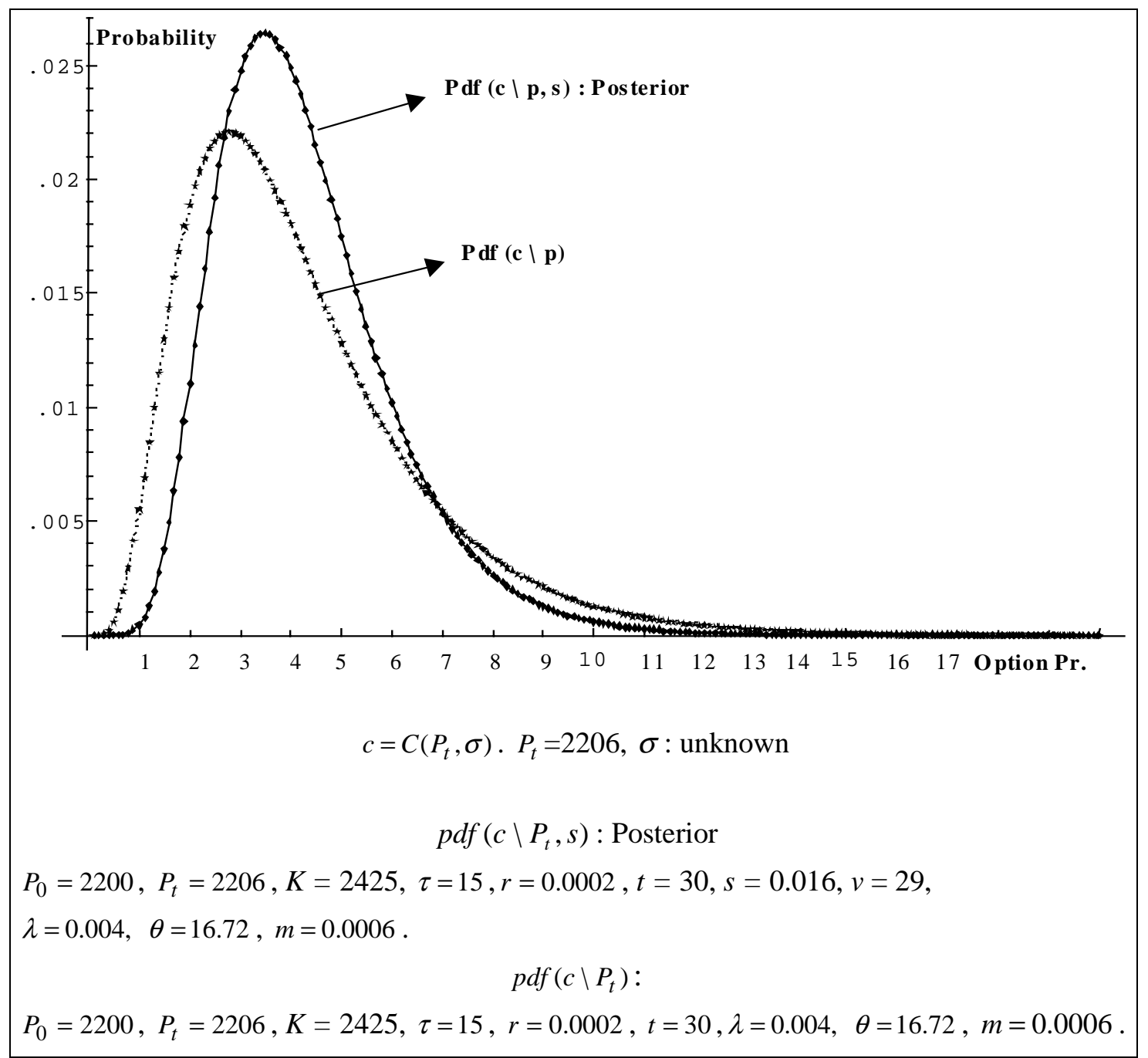

Figure 4.6. Posterior, and conditional (on the asset price) probability density functions for the BS option price. Out-of-the-money case.

Table 4.5.

Summary statistics of distributions exhibited in Figure 4.6

\begin{tabular}{lrrrrrc}
\hline Quantiles & 0.025 & 0.975 & Mean & Std. Dev. & Skewness & Exs. Kurtosis \\
\hline$p d f\left(c \backslash P_{t}, s\right):$ & 1.7 & 8.9 & 4.27 & 1.73 & 1.15 & 2.14 \\
\hline$p d f\left(c \backslash P_{t}\right):$ & 1.1 & 10.1 & 4.22 & 2.30 & 1.45 & 3.24 \\
\hline
\end{tabular}


Note that $p d f\left(c \backslash P_{t}, s\right)$ and $p d f\left(c \backslash P_{t}\right)$ are defined within the support of the distribution. For example for the case $K=2025$ the density has the support given by the no-arbitrage bounds of the option price: $187.066<c=C\left(P_{t}, \sigma\right)<2206$. For the cases $K=2225$ and $K=2425$, the support is $0<c=C\left(P_{t}, \sigma\right)<2206$. Also note that the distributions for an in-the-money and an out-of-the-money option are significantly more positively skewed and leptokurtic than the near-the-money (or at-the-money) cases (see Tables 4.3, 4.4, 4.5).

\section{Conclusion}

In the foregoing Bayesian analysis we have discussed the statistical properties of the Black-Scholes option price with the randomness arising from both the underlying asset price and its volatility. The results presented in Figures 4.1 - 4.6 and Tables 4.1 4.5 show the extent to which conditioning on the asset price dramatically reduces the variability of the option price. Indeed, since as a Bayesian problem the BS option price depends on both parameters (volatility: $(\sigma)$ ) and data (price: $\left(P_{t}\right)$ ), not conditioning on the data induces a very large dispersion in the option price. It should however be mentioned that as the conditioning horizon decreases (i.e. the time between the initial price $P_{0}$ and the final price $P_{t}$ ), variability in the option price gradually decreases as well. If we include the sample variance $\left(s^{2}\right)$ into the data, not conditioning on it, does not have as a dramatic impact as not conditioning on the asset price, but we show that conditioning on both results in less variability for the option price than conditioning only on price.

This will have important implications for forecasting. Although our paper is not about forecasting, we see our analysis as a necessary prelude to establishing a Bayesian theory of option price forecasting. Existing theories (e.g. Karolyi (1993), Noh, Engle and Kane (1994), Hwang and Satchell (1998), and many others) use only the implied volatility or other measures of volatility (e.g. GARCH)) to forecast option prices while keeping the price of the underlying fixed. Our theory will allow us to consider forecasting when both prices and volatility can vary.

Furthermore, our results have potential uses in risk management as we can report VaR (Value at Risk) and other distributional measures. Although we do not consider portfolio problems, it is possible to carry out such extensions. Likewise, one could use our methodology in option pricing models other than the Black-Scholes. Thus, at least in principle, we could incorporate randomness due to interest rates (Merton (1973)) or specific models of volatility (e.g. Duan (1995)). 


\section{Appendix}

A.1. Proposition 2.1: The joint unconditional density of the price $P_{t}$ and volatility $\sigma$ is given by:

$$
p d f\left(P_{t}, \sigma\right)=\frac{1}{\sqrt{\pi t} P_{t}} \frac{\lambda^{\theta}}{\Gamma(\theta)} \frac{1}{\sigma^{2(\theta+1)}} \exp \left(-\frac{\lambda}{\sigma^{2}}\right) \exp \left(-\frac{1}{4 \sigma^{2} t}\left[\ln \left(\frac{P_{t}}{P_{0}}\right)-\left(m-\frac{1}{2} \sigma^{2}\right) t\right]^{2}\right)
$$

\section{Proof of Proposition:}

From Assumptions 2.4 and 2.2 we have expressions for $p d f(\sigma)$ and $p d f(\mu \backslash \sigma)$ respectively. Then $p d f(\mu, \sigma)$ is just $p d f(\sigma)^{*} p d f(\mu \backslash \sigma)$ :

$$
p d f(\mu, \sigma)=\sqrt{\frac{2 t}{\pi}} \frac{\lambda^{\theta}}{\Gamma(\theta)} \frac{1}{\sigma^{2(\theta+1)}} \exp \left(-\frac{t(\mu-m)^{2}}{2 \sigma^{2}}\right) \exp \left(-\frac{\lambda}{\sigma^{2}}\right)
$$

Similarly $p d f\left(P_{t}, \mu, \sigma\right)$ is given by $p d f(\mu, \sigma) * p d f\left(P_{t} \backslash \mu, \sigma\right)$.

We have just obtained $p d f(\mu, \sigma)$ and in Assumption 2.1 we state $p d f\left(P_{t} \backslash \mu, \sigma\right)$.

Then

$p d f\left(P_{t}, \mu, \sigma\right)=$

$=\frac{1}{\pi P_{t}} \frac{\lambda^{\theta}}{\Gamma(\theta)} \frac{1}{\sigma^{2 \theta+3}} \exp \left(-\frac{\lambda}{\sigma^{2}}\right) \exp \left\{-\frac{1}{2 \sigma^{2} t}\left(\left[\ln \left(\frac{P_{t}}{P_{0}}\right)+\frac{\sigma^{2} t}{2}-\mu t\right]^{2}+t^{2}(\mu-m)^{2}\right)\right\}$.

To get the proposed result we therefore need to integrate out $\mu$ :

$$
\begin{aligned}
p d f\left(P_{t}, \sigma\right) & =\int_{-\infty}^{\infty} p d f\left(P_{t}, \mu, \sigma\right) \partial \mu \\
& =K \int_{-\infty}^{\infty} \exp \left\{-\frac{1}{2 \sigma^{2} t}\left((z-\mu t)^{2}+t^{2}(\mu-m)^{2}\right)\right\} \partial \mu
\end{aligned}
$$

where we have set $\quad K=\frac{1}{\pi P_{t}} \frac{\lambda^{\theta}}{\Gamma(\theta)} \frac{1}{\sigma^{2 \theta+3}} \exp \left(-\frac{\lambda}{\sigma^{2}}\right) \quad$ and $\quad z=\ln \left(\frac{P_{t}}{P_{0}}\right)+\frac{1}{2} \sigma^{2} t$.

Let us now evaluate the integral:

$$
\int_{-\infty}^{\infty} \exp \left\{-\frac{(z-\mu t)^{2}+t^{2}(\mu-m)^{2}}{2 \sigma^{2} t}\right\} \partial \mu
$$




$$
\begin{aligned}
& =\int_{-\infty}^{\infty} \exp \left(-\frac{1}{2 \sigma^{2} t}\left[\left(z^{2}+t^{2} m^{2}\right)+\mu^{2}\left(2 t^{2}\right)-2 \mu t(z+m t)\right] \partial \mu\right. \\
& =\int_{-\infty}^{\infty} \exp \left(-\frac{1}{2 \sigma^{2} t}\left[z^{2}+t^{2} m^{2}+2 t^{2}\left\{\mu^{2}-2 \mu\left(\frac{z+m t}{2 t}\right)+\left(\frac{z+m t}{2 t}\right)^{2}\right\}-2 t^{2}\left(\frac{z+m t}{2 t}\right)^{2}\right] \partial \mu\right. \\
& =\int_{-\infty}^{\infty} \exp \left(-\frac{1}{2 \sigma^{2} t}\left[\frac{1}{2}(z-t m)^{2}+2 t^{2}\left(\mu-\frac{z+m t}{2 t}\right)^{2} \partial \mu\right.\right. \\
& =\exp \left(-\frac{1}{4 \sigma^{2} t}(z-t m)^{2}\right)_{-\infty}^{\infty} \exp \left(-\frac{t}{\sigma^{2}}\left(\mu-\frac{z+m t}{2 t}\right)^{2}\right) \mu \\
& =\exp \left(-\frac{1}{4 \sigma^{2} t}(z-t m)^{2}\right) \sqrt{2 \pi}\left(\frac{\sigma}{\sqrt{2 t}}\right)\left[\frac{1}{\sqrt{2 \pi}\left(\frac{\sigma}{\sqrt{2 t}}\right)} \int_{-\infty}^{\infty} \exp \left(-\frac{1}{2\left(\sigma^{2} / 2 t\right)}\left(\mu-\frac{z+m t}{2 t}\right)^{2}\right) \mu\right] \\
& =\exp \left(-\frac{1}{4 \sigma^{2} t}(z-t m)^{2}\right)\left(\sqrt{\frac{\pi}{t}}\right) \sigma
\end{aligned}
$$

Therefore:

$p d f\left(P_{t}, \sigma\right)=K \exp \left(-\frac{1}{4 \sigma^{2} t}(z-t m)^{2}\right)\left(\sqrt{\frac{\pi}{t}}\right) \sigma$

Substituting in the values for $K$ and $z$ we get the proposed result for $p d f\left(P_{t}, \sigma\right)$.

A.2. Proposition 2.3: The unconditional density of the statistic $s$ is given by:

$$
p d f(s)=\frac{2}{\mathrm{~B}\left(\frac{v}{2}, \theta\right)} \lambda^{\theta}\left(\frac{v}{2}\right)^{\frac{v}{2}} \frac{s^{\nu-1}}{\left(\lambda+\frac{v s^{2}}{2}\right)^{\frac{v}{2}+\theta}} .
$$

\section{Proof of Proposition:}

$p d f\left(\sigma^{2}\right)$ is given in Assumption 2.4 and $p d f\left(s^{2} \backslash \sigma^{2}\right)$ in Assumption 2.3. 
Then: $p d f\left(s^{2}, \sigma^{2}\right)=p d f\left(\sigma^{2}\right) p d f\left(s^{2} \backslash \sigma^{2}\right)$

$=\frac{\lambda^{\theta}}{\Gamma(\theta)} \frac{v^{\frac{v}{2}}\left(s^{2}\right)^{\frac{v}{2}-1}}{2^{\frac{v}{2}} \Gamma\left(\frac{v}{2}\right)}\left(\frac{1}{\sigma^{2}}\right)^{\frac{v}{2}+\theta+1} \exp \left(-\frac{2 \lambda+v s^{2}}{2 \sigma^{2}}\right)$

Let $\quad K=\frac{\lambda^{\theta}}{\Gamma(\theta)} \frac{v^{\frac{v}{2}}\left(s^{2}\right)^{\frac{v}{2}-1}}{2^{\frac{v}{2}} \Gamma\left(\frac{v}{2}\right)}, \quad m=\frac{v}{2}+\theta+1, \quad a=\frac{2 \lambda+v s^{2}}{2}$.

Then $p d f\left(s^{2}\right)=\int_{0}^{\infty} p d f\left(s^{2}, \sigma^{2}\right) \partial \sigma^{2}=K \int_{0}^{\infty}\left(\frac{1}{\sigma^{2}}\right)^{m} \exp \left(-\frac{a}{\sigma^{2}}\right) \partial \sigma^{2}$

We need to evaluate:

$\int_{0}^{\infty}\left(\frac{1}{\sigma^{2}}\right)^{m} \exp \left(-\frac{a}{\sigma^{2}}\right) \partial \sigma^{2}$

Let $\quad \frac{1}{\sigma^{2}}=x \quad \Rightarrow \quad-\left(\frac{1}{\sigma^{2}}\right)^{2} \partial \sigma^{2}=\partial x$

$\therefore \quad \int_{0}^{\infty}\left(\frac{1}{\sigma^{2}}\right)^{m} \exp \left(-\frac{a}{\sigma^{2}}\right) \partial \sigma^{2}=-\int_{\infty}^{0} x^{m-2} \exp (-a x) \partial x=\int_{0}^{\infty} x^{m-2} \exp (-a x) \partial x$

Now

$\int_{0}^{\infty} x^{m-2} \exp (-a x) \partial x=\left[-\frac{1}{a} x^{m-2} \exp (-a x)\right]_{0}^{\infty}-\frac{m-2}{-a} \int_{0}^{\infty} x^{m-3} \exp (-a x) \partial x$

note that $\lim _{x \rightarrow \infty}\left(x^{k} e^{-x}\right)=0$

$=\frac{m-2}{a} \int_{0}^{\infty} x^{m-3} \exp (-a x) \partial x$

$=\frac{m-2}{a}\left\{\left[-\frac{1}{a} x^{m-3} \exp (-a x)\right]_{0}^{\infty}-\frac{m-3}{-a} \int_{0}^{\infty} x^{m-4} \exp (-a x) \partial x\right\}$

$=\frac{(m-2)(m-3)}{a^{2}} \int_{0}^{\infty} x^{m-4} \exp (-a x) \partial x=\ldots \ldots . .=\frac{(m-2) !}{a^{m-2}} \int_{0}^{\infty} \exp (-a x) \partial x$

$=-\frac{(m-2) !}{a^{m-1}}[\exp (-a x)]_{0}^{\infty}=\frac{(m-2) !}{a^{m-1}}$ 


$$
\begin{gathered}
=\frac{\left(\frac{v}{2}+\theta-1\right) !}{\left(\frac{2 \lambda+v s^{2}}{2}\right)^{\frac{v}{2}+\theta}} \quad\left(\text { remember } m=\frac{v}{2}+\theta+1, a=\frac{2 \lambda+v s^{2}}{2}\right) \\
\Rightarrow \quad p d f\left(s^{2}\right)=K^{*} \frac{\Gamma\left(\frac{v}{2}+\theta\right)}{\left(\lambda+\frac{v s^{2}}{2}\right)^{\frac{v}{2}+\theta}}
\end{gathered}
$$

Substituting for $K$ we get:

$$
\begin{aligned}
p d f\left(s^{2}\right) & =\frac{\lambda^{\theta}}{\Gamma(\theta)} \frac{v^{\frac{v}{2}}\left(s^{2}\right)^{\frac{v}{2}-1}}{2^{\frac{v}{2}} \Gamma\left(\frac{v}{2}\right)} \frac{\Gamma\left(\frac{v}{2}+\theta\right)}{\left(\lambda+\frac{v s^{2}}{2}\right)^{\frac{v}{2}+\theta}} \\
& =\frac{1}{\mathrm{~B}\left(\frac{v}{2}, \theta\right)} \lambda^{\theta}\left(\frac{v}{2}\right)^{\frac{v}{2}} \frac{\left(s^{2}\right)^{\frac{v}{2}-1}}{\left(\lambda+\frac{v s^{2}}{2}\right)^{\frac{v}{2}+\theta}} \cdot{ }^{19}
\end{aligned}
$$

Having obtained $p d f\left(s^{2}\right)$, it is straightforward to get $p d f(s)$ :

$$
p d f(s)=2 s^{*} p d f\left(s^{2}\right)=\frac{2}{\mathrm{~B}\left(\frac{v}{2}, \theta\right)} \lambda^{\theta}\left(\frac{v}{2}\right)^{\frac{v}{2}} \frac{s^{v-1}}{\left(\lambda+\frac{v s^{2}}{2}\right)^{\frac{v}{2}+\theta}} .
$$

${ }^{19}$ Note: This implies that $\frac{v s^{2}}{2}$ is unconditionally distributed Inverted-Beta-2:

$$
f_{i \beta 2}\left(\frac{v s^{2}}{2} \backslash \frac{v}{2}, \theta, \lambda\right) \equiv \frac{1}{\mathrm{~B}\left(\frac{v}{2}, \theta\right)} \lambda^{\theta} \frac{\left(\frac{v s^{2}}{2}\right)^{\frac{v}{2}-1}}{\left(\lambda+\frac{v s^{2}}{2}\right)^{\frac{v}{2}+\theta}} .
$$

where $f_{i \beta 2}(\ldots)$ denotes the Inverted-Beta-2 probability density function. 
A.3. Proposition 2.4: The marginal density for the asset price $P_{t}$ is given by:

$p d f\left(P_{t}\right)=$

$\frac{\lambda^{\theta} e^{-\frac{\ln \left(\frac{P_{t}}{P_{0}}\right)-m t}{4}}}{\sqrt{\pi t} P_{t} \Gamma(\theta)}\left(\frac{t}{16}\right)^{\theta+\frac{1}{2}}\left[\frac{4 \lambda t+\left(\ln \left(\frac{P_{t}}{P_{0}}\right)-m t\right)^{2}}{64}\right]^{-\frac{2 \theta+1}{4}} K_{\theta+\frac{1}{2}}\left(\theta+\frac{1}{2}, \frac{1}{4} \sqrt{4 \lambda t+\left(\ln \left(\frac{P_{t}}{P_{0}}\right)-m t\right)^{2}}\right)$

where $K_{\theta+\frac{1}{2}}\left(\frac{1}{4} \sqrt{4 \lambda t+\left(\ln \left(\frac{P_{t}}{P_{0}}\right)-m t\right)^{2}}\right)$ is the modified Bessel function of the second

kind of order $\theta+\frac{1}{2}$.

Proof of Proposition:

$$
p d f\left(P_{t}\right)=\int_{0}^{\infty} p d f\left(P_{t}, \sigma^{2}\right) \partial \sigma^{2}
$$

Note that

$$
p d f\left(P_{t}, \sigma^{2}\right)=\frac{1}{2 \sqrt{\pi t} P_{t}} \frac{\lambda^{\theta}}{\Gamma(\theta)}\left(\frac{1}{\sigma^{2}}\right)^{\theta+\frac{3}{2}} \exp \left(-\frac{\lambda}{\sigma^{2}}\right) \exp \left(-\frac{1}{4 \sigma^{2} t}\left[\ln \left(\frac{P_{t}}{P_{0}}\right)-\left(m-\frac{1}{2} \sigma^{2}\right) t\right]^{2}\right)
$$

(this follows straightforwardly from Proposition 2.1)

and let $A=\frac{1}{2 \sqrt{\pi t} P_{t}} \frac{\lambda^{\theta}}{\Gamma(\theta)} \quad$ and $\quad z=\ln \left(\frac{P_{t}}{P_{0}}\right)-m t$

Then $\quad p d f\left(P_{t}, \sigma^{2}\right)=A\left(\frac{1}{\sigma^{2}}\right)^{\left(\theta+\frac{1}{2}\right)+1} \exp \left(-\frac{\lambda}{\sigma^{2}}\right) \exp \left(-\frac{1}{4 \sigma^{2} t}\left[z-\frac{1}{2} \sigma^{2} t\right]^{2}\right)$

$$
\begin{aligned}
& =A\left(\frac{1}{\sigma^{2}}\right)^{\left(\theta+\frac{1}{2}\right)+1} \exp \left(-\frac{\lambda}{\sigma^{2}}\right) \exp \left(-\frac{z^{2}}{4 \sigma^{2} t}-\frac{z}{4}-\frac{\sigma^{2} t}{16}\right) \\
& =A \exp \left(-\frac{z}{4}\right)\left(\frac{1}{\sigma^{2}}\right)^{\left(\theta+\frac{1}{2}\right)+1} \exp \left[-\frac{\left(\lambda+z^{2} / 4 t\right)}{\sigma^{2}}\right] \exp \left(-\frac{t}{16} \sigma^{2}\right)
\end{aligned}
$$


Also let $\quad c=\lambda+\frac{z^{2}}{4 t} \quad$ and $\quad p=\frac{t}{16}$

Then

$$
\begin{aligned}
& p d f\left(P_{t}\right)=\int_{0}^{\infty} p d f\left(P_{t}, \sigma^{2}\right) \partial \sigma^{2}= \\
& A \exp \left(-\frac{z}{4}\right) \frac{\Gamma\left(\theta+\frac{1}{2}\right)}{c^{\theta+\frac{1}{2}}} \int_{0}^{\infty} \underbrace{\frac{c^{\theta+\frac{1}{2}}}{\Gamma\left(\theta+\frac{1}{2}\right)}\left(\frac{1}{\sigma^{2}}\right)^{\left(\theta+\frac{1}{2}\right)+1} \exp \left[-\frac{c}{\sigma^{2}}\right] \exp \left(-p \sigma^{2}\right) \partial \sigma^{2}}_{\text {Inverted-Gamma-1 function }}
\end{aligned}
$$

Observe now that the required integral is the Laplace transform of an InvertedGamma function:

$L\left(f_{i \gamma}\right)=F(p)=\int_{0}^{\infty} f_{i \gamma}\left(\sigma^{2}\right) e^{-p \sigma^{2}} d \sigma^{2}$.

Hence

$$
p d f\left(P_{t}\right)=A \exp \left(-\frac{z}{4}\right) 2 p^{\theta+1 / 2}(c p)^{-\frac{2 \theta+1}{4}} K_{\theta+\frac{1}{2}}(2 \sqrt{c p})
$$

Substituting in the values for $A, z, p$, and $c$ we get the proposed result.

\section{A.4.}

i) We want to derive the density of the option price conditional on the sample estimate of volatility: i.e. $p d f(c \backslash s)$.

Consider first $p d f\left(P_{t}, \sigma \backslash s\right)=\frac{p d f\left(P_{t}, \sigma, s\right)}{p d f(s)}$.

$p d f\left(P_{t}, \sigma, s\right)$ is given in Proposition 2.2 and $p d f(s)$ in Proposition 2.3. Hence

$$
\begin{aligned}
& p d f\left(P_{t}, \sigma \backslash s\right)= \\
& \frac{1}{\sqrt{\pi t} P_{t} \Gamma\left(\frac{v}{2}+\theta\right)} \frac{\left(\lambda+\frac{v s^{2}}{2}\right)^{\frac{v}{2}+\theta}}{\sigma^{2 \theta+v+2}} \exp \left(-\frac{2 \lambda+v s^{2}}{2 \sigma^{2}}\right) \exp \left(-\frac{1}{4 \sigma^{2} t}\left[\ln \left(\frac{P_{t}}{P_{0}}\right)-\left(m-\frac{1}{2} \sigma^{2}\right) t\right]^{2}\right)
\end{aligned}
$$


Using now the same transformation as we did for the prior density:

$c=C\left(P_{t}, \sigma\right) \Rightarrow \Psi(c)=\Psi(c, \sigma)=P_{t}$

$\sigma=\sigma$

we get

$p d f(c, \sigma \backslash s)=$

$\frac{1}{\sqrt{\pi} \Psi(c, \sigma) \Gamma\left(\frac{v}{2}+\theta\right)} \frac{\left(\lambda+\frac{v s^{2}}{2}\right)^{\frac{v}{2}+\theta}}{\sigma^{2 \theta+v+2}} \exp \left(-\frac{2 \lambda+v s^{2}}{2 \sigma^{2}}\right) \exp \left(-\frac{1}{4 \sigma^{2} t}\left[\ln \left(\frac{\Psi(c, \sigma)}{P_{0}}\right)-\left(m-\frac{1}{2} \sigma^{2}\right) t\right]^{2}\right) \frac{1}{\Phi\left(d_{1}^{*}\right)}$

where $d_{1}^{*}=\frac{\left[\ln \left(\frac{\Psi(c, \sigma)}{K e^{-r \tau}}\right)+\frac{\sigma^{2} \tau}{2}\right]}{\sigma \sqrt{\tau}}$

Integrating out $\sigma$ numerically will give us $p d f(c \backslash s)$.

ii) We also want to derive the density of the option price conditional on the price: i.e. $p d f\left(c \backslash P_{t}\right)$.

Consider first $p d f\left(\sigma \backslash P_{t}\right)=\frac{p d f\left(P_{t}, \sigma\right)}{p d f\left(P_{t}\right)}$.

$p d f\left(P_{t}, \sigma\right)$ is given in Proposition 2.1 and $p d f\left(P_{t}\right)$ in Proposition 2.4.

Applying the transformation:

$c=C(\sigma) \Leftrightarrow \sigma=\Theta(c)$

we get:

$p d f\left(c \backslash P_{t}\right)=p d f\left(\Theta(c) \backslash P_{t}\right) * \operatorname{veg} a=$

$$
\frac{\frac{1}{\Theta[c]^{2(\theta+1)}} \exp \left(-\frac{\lambda}{\Theta[c]^{2}}\right) \exp \left(-\frac{1}{4 \Theta[c]^{2} t}\left[\ln \left(\frac{P_{t}}{P_{0}}\right)-\left(m-\frac{1}{2} \Theta[c]^{2}\right) t\right]^{2}\right) \exp \left(\left[\ln \left(\frac{P_{t}}{P_{0}}\right)-m t\right] / 4\right)}{\left(\frac{t}{16}\right)^{\theta+\frac{1}{2}}\left[\frac{4 \lambda t+\left(\ln \left(\frac{P_{t}}{P_{0}}\right)-m t\right)^{2}}{64}\right]^{-\frac{2 \theta+1}{4}} K_{\theta+\frac{1}{2}}\left(\theta+\frac{1}{2}, \frac{1}{4} \sqrt{4 \lambda t+\left(\ln \left(\frac{P_{t}}{P_{0}}\right)-m t\right)^{2}}\right)} * \text { vega }
$$




\section{References}

Appostol, T. (1969): Calculus: Multi-Variable Calculus and Linear Algebra, with Applications to Differential Equations and Probability, Volume II, John Wiley \& Sons.

Arrow, K., and A. Fisher (1974): "Environmental Preservation Uncertainty, and Irreversibility," Quarterly Journal of Economics, 88, 312-319.

Black, F. (1976): "Studies of Stock Price Volatilities," Proceedings of the 1976 Meetings of the American Statistical Association, Business and Economics Section, $56,177-181$.

Black, F. and M. Scholes (1973): "The Pricing of Options and Corporate Liabilities," Journal of Political Economy, 81, 637-659.

Bollerslev, T. (1986): "Generalised Autoregressive Conditional Heteroskedasticity," Journal of Econometrics, 31, 307-327.

Boyle, P., and A. Ananthanarayanan (1977): "The Impact of Variance Estimation in Option Valuation Models," Journal of Financial Economics, 5, 375-387.

Butler, J., and B. Schachter (1986): "Unbiased Estimation of the Black/Scholes Formula," Journal of Financial Economics, 15, 341-357.

Chiras, D.P., and S. Manaster (1978): "The Information Content of Option Prices and a Test of Market Efficiency," Journal of Financial Economics, 6, 213-234.

Christie, A. (1982): "The Stochastic Behaviour of Common Stock Variances," Journal of Financial Economics, 10, 407-432.

Day, R., and C. Lewis (1988): "The Behaviour of the Volatility Implicit in the Prices of Stock Index Options," Journal of Financial Economics, 22, 103-122.

Day, R., and C. Lewis (1992): "Stock Market Volatility and the Information Content of Stock Index Options," Journal of Econometrics, 52, 267-287.

Dixit, A. (1989): "Entry and Exit Decisions under Marketing," Journal of Political Economy, 97, 621-638.

Duan, J. (1995): " The GARCH option pricing model," Mathematical Finance, 5(1), 13-32. 
Efron B., and C. Morris (1973): "Stein's Estimation Rule and Its Competitors-An Empirical Bayes Approach," Journal of the American Statistical Association, 68, 117 130.

Engle, R.F. (1982): "Autoregressive Conditional Heteroskedasticity with Estimates of the Variances of U.K. Inflation," Econometrica, 50, 987-1008.

Engle, R.F., and T. Bollerslev (1986): "Modelling Persistence of Conditional Variances," Econometric Reviews, 5, 1-50.

Engle, R.F., and C. Mustafa (1992): "Implied ARCH Models from Option Prices," Journal of Econometrics, 52, 289-311.

Epps, T., and M. Epps (1976): "The Stochastic Dependence of Security Price Changes and Transactions Volume: Implications for the Mixture of Distributions Hypothesis," Econometrica, 44, 305-321.

French, K.R., G.W. Schwert, and R. Stambaugh (1987): "Expected Stock Returns and Volatility," Journal of Financial Economics, 19, 3-29.

Garman, M., and M. Klass (1980): "On Estimation of Security Price Volatility from Historical Data," Journal of Business, 53, 67-78.

Ghysels, E., A. Harvey, and E. Renault (1996): "Stochastic Volatility," in G.S. Maddala and C.R. Rao Eds., Handbook of Statistics, Vol. 14. Amsterdam, New York, and Oxford: Elsevier, Norh-Holland.

Hull, J., and A. White (1987): "The Pricing of Options on Assets with Stochastic Volatilities," Journal of Finance, 42, 281-300.

Hutchinson, J., A. Lo, and T. Poggio (1994): "A NonParametric Approach to the Pricing and Hedging of Derivative Securities Via Learning Networks," Journal of Finance, 49, 851-889.

Hwang, S., and S. Satchell (1998): "Implied Volatility Forecasting: a comparison of different procedures including fractionally integrated models with applications to UK equity options," in J. Knight and S. Satchell Eds., Forecasting Volatility in the Financial Markets, Butterworth-Heinemann.

Jackwerth, J., and M. Rubinstein (1996): "Recovering Probability Distributions from Option Prices," Journal of Finance, 1611-1631. 
Karolyi, G.A. (1993): "A Bayesian Approach to Modelling Stock Return Volatility for Option Valuation," Journal of Financial and Quantitative Analysis, 28, 579-594.

Kemp, M. (1996): Actuaries and Derivatives. Paper prepared for the Institute of Actuaries, London, 28 October.

Knight, J., and S. Satchell (1997): "Existence of Unbiased Estimators of the Black/Scholes Option Price, Other Derivatives, and Hedge Ratios," Econometric Theory, 13, 791-807.

Latane, H., and R. Rendleman (1976): "Standard Deviation of Stock Prices Implied in Option Prices," Journal of Finance, 31, 369-381.

McDonald, R., and D. Siegel (1985): "Investment and the Valuation of Firms when there is an Option to Shut Down," International Economic Review, 26, 331-349.

McDonald, R., and D. Siegel (1986): "The Value of Waiting to Invest," Quarterly Journal of Economics, 106, 707-727.

Melino, A., and S.M. Turnbull (1990): "Pricing Foreign Currency Options with Stochastic Volatility," Journal of Econometrics, 45, 239-265.

Merton, R. (1973): "Theory of Rational Option Pricing," Bell Journal of Economics and Management Science, 4, 141-83.

Morgan, I. (1976): "Stock Prices and Heteroscedasticity," Journal of Business, 49, 496-508.

Ncube M., and S. Satchell (1997): "The Statistical Properties of the Black-Scholes Option Price," Mathematical Finance, 7, 287-305.

Nelson, D.B. (1991): "Conditional Heteroscedasticity in Asset Returns: A New Approach," Econometrica, 59, 347-370.

Noh, J., F. Engle, and A. Kane (1994): "Forecasting Volatility and Option Prices of the S\&P 500 Index," Journal of Derivatives, 17-30.

Parkinson, M. (1980): "The Extreme Value Method of Estimating the Variance of the Rate of Return," Journal of Business, 53, 61-66. 
Poterba, J., and L. Summers (1987): "The Persistence of Volatility and Stock Market Fluctuations," American Economic Review, 76, 1142-1151.

Pyle, D. (1995): U.S. savings and loan crisis. In R. Jarrow, V. Maksimowe, and W. Ziwmba (eds.), Handbook in Operations Research and Management Science, Vol. 19: Finance. Amsterdam: North-Holland.

Rubinstein, M. (1994): "Implied Binomial Trees," Journal of Finance, 49, 771-818. Scott, L. (1991): "Random Variance Option Pricing," Advances in Futures and Options Research, 5, 113-135.

Tauchen, G., and M. Pitts (1983): "The Price Variability-Volume Relationship on Speculative Markets," Econometrica, 51, 485-505.

Wiggins, J. (1987): "Option Values under Stochastic Volatility: Theory and Empirical Estimates," Journal of Financial Economics, 19, 351-372.

Zellner, A. (1971): An introduction to Bayesian Inference in Econometrics, Wiley, New York. 